\title{
Association Between Cystatin C, Cystatin C Rangeability and Mortality of COVID-19 Patients With or Without Type 2 Diabetes Mellitus: An Opportunistic Retrospective Analysis
}

\section{Lei Yang}

The First Affiliated Hospital of xi'an Jiaotongl University

\section{Dou Xu}

Xi'an Jiaotong University, school of software engineering

\section{Yiqing Tan}

Wuhan Third Hosipital

Bolin Li

The First Affiliated Hospital of Xi'an Jiaotong university

Dan Zhu

Shanghai 9th Peoples Hospital Affiliated to Shanghai Jiaotong University School of Medicine Jingbo Wang

Shanghai 9th Peoples Hospital Affiliated to Shanghai Jiaotong University School of Medicine Hui Sun

Sense Time Research

\section{Xinglong Liu}

Sense Time Research

\section{Xiao-Pu Zheng}

The First Affiliated Hospital of Xi'an Jiaotong University

\section{Ling Zhu}

Shanghai 9th Peoples Hospital Affiliated to Shanghai Jiaotong University School of Medicine

Zhongyu Li ( $\square$ zhongyuli@xjtu.edu.cn )

Xi'an Jiaotong University School of Software Engineering

\section{Original investigation}

Keywords: COVID-19, Cystatin C, Renal function, All-cause death, Type 2 diabetes mellitus

Posted Date: November 24th, 2020

DOI: https://doi.org/10.21203/rs.3.rs-112220/v1 
License: (c) (i) This work is licensed under a Creative Commons Attribution 4.0 International License. Read Full License 


\section{Abstract}

Background: Since December of 2019, novel coronavirus (SARS-CoV-2)-induced pneumonia (COVID-19) exploded in Wuhan, and rapidly spread throughout China. Patients with COVID-19 demonstrated quite different appearances and outcomes in clinical manifestations. We aimed to figure out whether risk factors of the cystatin $\mathrm{C}$ (CysC) and the CysC rangeability are influencing the prognosis of COVID-19 patients with or without type 2 diabetes mellitus (T2DM).

Methods: 675 T2DM patients and 602 non-T2DM patients were divided into low CysC group, high CysC group and low CysC rangeability group, high CysC rangeability group according to the serum CysC level and the change range of CysC. Demographic characteristics, clinical data and laboratory results of the four groups were collected and analyzed.

Results: Our data showed that COVID-19 patients with high CysC level and CysC rangeability had more organic damage and higher mortality rate compared to those with low level or low rangeability of CysC. Furthermore, patients with higher CysC level and $\mathrm{CysC}$ rangeability also demonstrated higher blood lymphocytes (lymph), C-reactive protein (CRP), alanine aminotransferase (ALT), aspartate aminotransferase (AST) which may greatly influence disease progression and poor prognosis of COVID19. After adjusting for possible confounders, multivariate analysis revealed that $\mathrm{CysC} \leq 0.93 \mathrm{mg} / \mathrm{dl}$ as a reference, CysC $\varangle 0.93 \mathrm{mg} / \mathrm{dl}$ were significantly associated with the risk of heart failure $(\mathrm{OR}=2.401,95 \% \mathrm{Cl}$ : $1.118-5.156)$ and all-cause death $(\mathrm{OR}=2.734,95 \% \mathrm{Cl}$ : $1.098-6.811)$; referring to CysC rangeability $\leq 0$, CysC rangeability $\mathbb{0}$ significantly associated with all-cause death (OR=4.029, 95\% Cl: 1.864-8.706). Further grouped by T2DM, these associations were stronger in T2DM than in non-T2DM.

Conclusions: It suggests that $\mathrm{CysC}$ level and $\mathrm{CysC}$ rangeability contribute to clinical manifestations and may influence the prognosis of COVID-19. The CysC is considered as a potential risk factor of the prognosis of COVID-19. Special medical care and appropriate intervention should be performed in COVID19 patients with elevated CysC during hospitalization and later clinical follow-up, especially for those with T2DM.

\section{Introduction}

In late December 2019, in Wuhan City of China, many patients with undefined pneumonia were reported to be infected with a novel coronavirus (SARS-CoV-2), formally named coronavirus disease 2019 (COVID19) by the World Health Organization (WHO) ${ }^{1-3}$. COVID-19 can be mainly spread by respiratory droplets and contact, aerosol transmission, as well as fecal-oral transmission ${ }^{4}$. As with highly infectious, this disease is listed as a class B infectious disease, recommended preventive and control measures similar to those against class A infectious diseases by "the law of the People's Republic of China on the prevention and treatment of infectious diseases". The epidemic continues and the population is expanding, which has brought great challenges to all countries in the world. 
The clinical characteristics of covid-19 patients, such as male, older age and potential complications, may lead to increased ICU admission and mortality. Particularly those with pre-existing conditions, diabetes, heart disease ${ }^{5}$, acute respiratory distress syndrome (ARDS), acute renal injury, liver injury, shock, and multiorgan failure, can be considered as the characteristic of critical patients ${ }^{6,7}$. Therefore, it is critical to pay special attention to the potential organ injuries and comorbidities for the clinical implementation of therapeutic measures ${ }^{7}$.

So far, the acute kidney injury (AKI) has been observed in COVID-19 patients, with a general incidence ranging from $3-15 \%{ }^{7}$, and up to $25 \%$ in critically-ill patients ${ }^{8}$. Meanwhile, AKI was demonstrated to be with high probability for severe ARDS, which requires invasive mechanical ventilation, especially for older or those with comorbidities such as hypertension or $\mathrm{T}_{2} \mathrm{DM}^{9}$. Recent studies suggested that acute renal damage can frequently occur in earlier stages of COVID-19 disease, which also associates with mortality ${ }^{10-12}$.

Preexisted renal disease may also associate with an increased risk for adverse outcomes, with an adjusted hazard ratio of $2.0(95 \% \mathrm{Cl}, 1.32$ to 3.15$)$ for hospitalized patients ${ }^{13}$. Although this evidence emphasizes that $\mathrm{AKI}$ and severe kidney diseases are independent risk factors for poor prognosis of COVID-19 disease, based on the rapid development of urea nitrogen and creatinine. The contribution of the $\mathrm{CysC}$ and the changes of CysC with respect to the disease on outcomes of COVID-19 patients are still poorly understood.

As a super-aging country, China has an increasing population of T2DM, which were more likely to develop COVID-19 and worse clinical outcomes, in comparison with the non-T2DM of COVID-19 patients ${ }^{14}$. Therefore, we focused on COVID-19 patients with T2DM and investigated the association between CysC and changes of CysC concerning clinical outcomes. Moreover, we also investigated the effect of modification of T2DM on those associations. We hypothesized that $\mathrm{Cys} C$ and changes of CysC are associated with worse organ function, higher risk of death in COVID-19 patients.

\section{Methods}

\section{Study Population and Data Collection}

This is a retrospective study conducted in Wuhan Third Hospital. The study was approved by the Ethics Committees of the hospital. We considered all consecutive severe patients with COVID-19 who were admitted to the hospital between the date from January 11 to March 1, 2020. All patients were diagnosed based on the recommendations by the National Institute for Viral Disease Control and Prevention of China (5th edition), by specifying suspected cases who have contact history with diagnosed COVID-19 patients, as well as presenting typical chest CT features. A positive PCR (Polymerase Chain Reaction) test for SARS-CoV-2 is mandatory for patients to make the diagnosis. Demographic characteristics, clinical information, laboratory test results, as well as clinical outcome data, were obtained using data collection 
forms from electronic medical records. The duration from the onset of disease to hospital admission and death was also recorded.

\section{Definition}

We assessed the impact of kidney injury severity on admission and changes in renal function during the course of disease were on the prognosis of 1277 patients with COVID-19. Kidney injury severity was measured by $\mathrm{CysC}$ level on admission, where the renal function changes were defined as CysC rangeability, calculated as the difference of $\mathrm{CysC}$ level between the time of admission and the highest CysC level testing during hospitalization. We classified patients into T2DM and non-T2DM group to eliminate the affection of T2DM itself on kidney function. Diabetics and non-diabetics were hierarchically classified into 2 groups according to the median value of CysC on admission and CysC rangeability respectively. Severe inflammation was defined as highest neutrophil-to-lymphocyte ratio (NLR) during hospitalization higher than 10. Liver injury was defined as ALT or AST at any time during hospitalization greater than $80 \mathrm{U} / \mathrm{L}$. Heart failure was defined as highest brain natriuretic peptide (BNP) during hospitalization greater than $300 \mathrm{pg} / \mathrm{ml}$.

\section{Statistical Analysis}

Continuous variables are presented as the mean \pm standard deviation if normally distributed or median (lower quartile, upper quartile) otherwise, and Shapiro-Wilk test was used for normality test. Categorical variables are presented as numbers and percentages. Differences in the parameters among groups were analyzed using student's t-test for normally distributed variables, the Mann-Whitney $U$ test was used for non-normally distributed continuous variables, and the chi-square test was used for categorical variables. Logistic regression models were used in univariate analyses and multivariate analyses to determine the prognostic value of CysC. Multivariate analyses adjusted for significant baseline variables and the factors closely related to the outcome of patients with cardiovascular disease, such as age, sex, severe pneumonia, serum albumin, blood glucose, Lg BNP.

\section{Results}

\section{Characteristics of hospitalized patients with COVID-19}

A total of 1277 COVID-19-associated hospitalized adults from Wuhan city were included. Among the 1277 hospitalized adults, the median age was 63 years, (interquartile range [IQR], 51-70) (Table 1). 9.4\% of COVID-19 patients were diagnosed with severe pneumonia and $48 \%$ were males. There were 675 patients with T2DM and 602 patients with no T2DM. 8.5\% and $10.2 \%$ severe pneumonia were diagnosed in non-T2DM and T2DM patients respectively (Table 1). 
Table 1

characteristics of patients grouped by T2DM

\begin{tabular}{|c|c|c|c|c|}
\hline Variables & $\begin{array}{l}\text { Total } \\
(n=1277)\end{array}$ & $\begin{array}{l}\text { Non-T2DM } \\
(n=602)\end{array}$ & $\begin{array}{l}\text { T2DM } \\
(n=675)\end{array}$ & $\mathbf{p}$ \\
\hline Age & $63(51,70)$ & $66(55,72)$ & $61(47.5,68)$ & $\begin{array}{l}< \\
0.001\end{array}$ \\
\hline Males & $609(48)$ & $281(47)$ & $328(49)$ & 0.53 \\
\hline Severe pneumonia, $\mathrm{n}(\%)$ & $120(9.4)$ & $51(8.5)$ & $69(10.2)$ & 0.285 \\
\hline CysC baseline, mg/L & $0.93(0.77,1.14)$ & $0.96(0.79,1.19)$ & $0.9(0.74,1.12)$ & $\begin{array}{l}< \\
0.001\end{array}$ \\
\hline LgBNP, pg/mL & $1.29(0.53,1.71)$ & $1.4(0.77,1.8)$ & $1.21(0.38,1.66)$ & $\begin{array}{l}<.001 \\
0.001\end{array}$ \\
\hline Lymph_baseline, $10^{9} / \mathrm{L}$ & $1.15(0.82,1.6)$ & $1.19(0.86,1.62)$ & $1.1(0.8,1.57)$ & 0.044 \\
\hline Neutrop_baseline, $10^{9} / \mathrm{L}$ & $3.24(2.39,4.51)$ & $3.37(2.54,4.64)$ & $3.1(2.25,4.41)$ & 0.001 \\
\hline NLR baseline & $2.75(1.80,4.43)$ & $2.50(1.69,3.69)$ & $3.60(2.34,6.05)$ & $\begin{array}{l}<.001 \\
0.001\end{array}$ \\
\hline PCT_baseline, ug/L & $0.03(0.03,0.05)$ & $0.03(0.03,0.04)$ & $0.03(0.03,0.05)$ & 0.884 \\
\hline ALT_baseline, units/L & $24(16,37)$ & $22(15,35)$ & $26(16,39)$ & 0.003 \\
\hline AST baseline, units/L & $26(20,37)$ & $25(19,34)$ & $27(21,39)$ & 0.034 \\
\hline ALB_baseline, $\mathrm{g} / \mathrm{L}$ & $37.42 \pm 5.07$ & $37.47 \pm 5.13$ & $37.38 \pm 5.02$ & 0.765 \\
\hline Glu_baseline, mmol/L & $5.47(4.73,6.92)$ & $5.25(4.65,6.58)$ & $5.68(4.86,7.2)$ & $\begin{array}{l}<.001 \\
0.001\end{array}$ \\
\hline Cre_baseline, umol/L & $63(51.3,78.2)$ & $60.7(50,78.4)$ & $64.7(52.42,78.05)$ & 0.09 \\
\hline ALP_baseline, units/L & $60(49,75)$ & $63(51,78)$ & $59(48,73)$ & 0.004 \\
\hline CK_baseline, units/L & $67(45,116)$ & $65(44,107)$ & $71(46,124.25)$ & 0.021 \\
\hline CK-MB_baseline, ng/mL & $10(7,14)$ & $10(7,13)$ & $10(8,14)$ & 0.061 \\
\hline cTnl_baseline, ug/L & $0(0,0.01)$ & $0(0,0.01)$ & $0(0,0.01)$ & 0.369 \\
\hline hsCRP_baseline, mg/L & $12.5(2.2,20)$ & $7.1(1.9,20)$ & $20(2.89,20)$ & $\begin{array}{l}<.001 \\
0.001\end{array}$ \\
\hline \multicolumn{5}{|c|}{$\begin{array}{l}\text { Data are reported as mean } \pm \text { SD, median (IQR) or number and percentage. T2DM, Type } 2 \text { diabetes } \\
\text { mellitus; BNP, Brain Natriuretic Peptide; NLR, Neutrophil-to-Lymphocyte Ratio; PCT, procalcitonin; ALT, } \\
\text { Alanine aminotransferase; AST, Aspartate aminotransferase; ALB, albumin; Glu, glucose; Cre, } \\
\text { creatinine; ALP, alkaline phosphatase; CK, choline kinase; CK-MB, creatine kinase isoenzymes; cTnl, } \\
\text { hypersensitive troponin l; hsCRP, high-sensitivity C-reactive protein; DD, D-Dimer; Lymph, lymphocyte; } \\
\text { Neutrop, Neutrophil; CRP, C-reactive protein; PCT, procalcitonin; ALT, alanine transaminase; CySC, } \\
\text { Cystatin C. }\end{array}$} \\
\hline
\end{tabular}




\begin{tabular}{|c|c|c|c|c|}
\hline Variables & $\begin{array}{l}\text { Total } \\
(n=1277)\end{array}$ & $\begin{array}{l}\text { Non-T2DM } \\
(n=602)\end{array}$ & $\begin{array}{l}\text { T2DM } \\
(n=675)\end{array}$ & $\mathbf{p}$ \\
\hline DD_baseline, mg/L & $0.63(0.35,1.37)$ & $0.69(0.39,2.15)$ & $0.57(0.33,1.1)$ & $\begin{array}{l}< \\
0.001\end{array}$ \\
\hline Peak Lymph, $10^{9} / \mathrm{L}$ & $1.52(1.19,1.92)$ & $1.47(1.11,1.91)$ & $1.54(1.25,1.93)$ & 0.152 \\
\hline Peak Neutrop, $10^{9} / \mathrm{L}$ & $4.75(3.29,7.54)$ & $4.73(3.40-7.01)$ & $4.76(3.19,7.87)$ & 0.065 \\
\hline Peak NLR & $1.00(0.78,3.75)$ & $2.06(1.57,5.77)$ & $1.00(0.87,2.55)$ & 0.001 \\
\hline Peak CRP, mg/L & $\begin{array}{l}32.85 \text { (6.76, } \\
99.93)\end{array}$ & $\begin{array}{l}15.48(3.56 \\
71.52)\end{array}$ & $\begin{array}{l}56.58(10.96 \\
150.96)\end{array}$ & $\begin{array}{l}< \\
0.001\end{array}$ \\
\hline Peak PCT, ug/L & $0.38(0.13,2.5)$ & $0.26(0.1,2.46)$ & $0.48(0.15,2.58)$ & 0.374 \\
\hline Peak ALT, units/L & $46(27,82)$ & $42(24,69.25)$ & $48(28,91)$ & 0.053 \\
\hline Peak AST, units/L & $35(24,64)$ & $33(24,56)$ & $39(25,68.75)$ & 0.062 \\
\hline Peak ALB, g/L & $38.64 \pm 4.05$ & $38.59 \pm 4.19$ & $38.69 \pm 3.93$ & 0.791 \\
\hline Peak Cre, umol/L & $67.1(54.5,84.35)$ & $65.2(53.65,80.3)$ & $69(55.65,94.85)$ & 0.053 \\
\hline Peak ALP, units/L & $74(60,93)$ & $76(63,94)$ & $73(59,91)$ & 0.108 \\
\hline Peak CK, units/L & $71(47.5,210.5)$ & $63(44.5,173)$ & $85(53.75,246.5)$ & 0.116 \\
\hline Peak CK-MB, ng/mL & $14(10,23)$ & $13(10,21)$ & $14(10,26.25)$ & 0.386 \\
\hline Peak cTnl, ug/L & $0.08(0.02,0.48)$ & $0.05(0.02,0.7)$ & $0.09(0.02,0.42)$ & 0.644 \\
\hline Peak CysC, mg/L & $1.19(0.99,1.54)$ & $1.21(0.98,1.57)$ & $1.17(0.99,1.51)$ & 0.755 \\
\hline Peak hs-CRP, mg/L & $7.61(2.03,20)$ & $6.28(2.02,20)$ & $20(2.35,27)$ & 0.243 \\
\hline Peak DD, mg/L & $3.14(0.97,6.02)$ & $2.96(0.9,6.32)$ & $3.43(0.98,5.88)$ & 0.631 \\
\hline Hospital stays (days) & $15(11,17)$ & $16(14,17)$ & $14(11,17)$ & 0.003 \\
\hline Liver injury, n (\%) & & & & 0.005 \\
\hline 0 (no) & $1144(90)$ & $555(92)$ & $589(87)$ & \\
\hline 1 (yes) & $133(10)$ & $47(8)$ & $86(13)$ & \\
\hline Heart failure, n (\%) & & & & 0.318 \\
\hline \multicolumn{5}{|c|}{$\begin{array}{l}\text { Data are reported as mean } \pm \text { SD, median (IQR) or number and percentage. T2DM, Type } 2 \text { diabetes } \\
\text { mellitus; BNP, Brain Natriuretic Peptide; NLR, Neutrophil-to-Lymphocyte Ratio; PCT, procalcitonin; ALT, } \\
\text { Alanine aminotransferase; AST, Aspartate aminotransferase; ALB, albumin; Glu, glucose; Cre, } \\
\text { creatinine; ALP, alkaline phosphatase; CK, choline kinase; CK-MB, creatine kinase isoenzymes; cTnl, } \\
\text { hypersensitive troponin l; hsCRP, high-sensitivity C-reactive protein; DD, D-Dimer; Lymph, lymphocyte; } \\
\text { Neutrop, Neutrophil; CRP, C-reactive protein; PCT, procalcitonin; ALT, alanine transaminase; CysC, } \\
\text { Cystatin C. }\end{array}$} \\
\hline
\end{tabular}




\begin{tabular}{|c|c|c|c|c|}
\hline Variables & $\begin{array}{l}\text { Total } \\
(n=1277)\end{array}$ & $\begin{array}{l}\text { Non-T2DM } \\
(n=602)\end{array}$ & $\begin{array}{l}\text { T2DM } \\
(n=675)\end{array}$ & $\mathbf{p}$ \\
\hline 0 (no) & $1232(96)$ & $577(96)$ & $655(97)$ & \\
\hline 1 (yes) & $45(4)$ & $25(4)$ & $20(3)$ & \\
\hline $\begin{array}{l}\text { Severe inflammatory, } \mathrm{n} \\
(\%)\end{array}$ & & & & $\dot{0.001}$ \\
\hline 0 (no) & $1162(91)$ & $521(87)$ & $641(95)$ & \\
\hline 1 (yes) & $115(9)$ & $81(13)$ & $34(5)$ & \\
\hline All-cause death, n (\%) & & & & $\begin{array}{l}< \\
0.001\end{array}$ \\
\hline 0 (no) & $1221(96)$ & $602(100)$ & $619(92)$ & \\
\hline 1 (yes) & $56(4)$ & $0(0)$ & $56(8)$ & \\
\hline \multicolumn{5}{|c|}{$\begin{array}{l}\text { Data are reported as mean } \pm \text { SD, median (IQR) or number and percentage. T2DM, Type } 2 \text { diabetes } \\
\text { mellitus; BNP, Brain Natriuretic Peptide; NLR, Neutrophil-to-Lymphocyte Ratio; PCT, procalcitonin; ALT, } \\
\text { Alanine aminotransferase; AST, Aspartate aminotransferase; ALB, albumin; Glu, glucose; Cre, } \\
\text { creatinine; ALP, alkaline phosphatase; CK, choline kinase; CK-MB, creatine kinase isoenzymes; cTnl, } \\
\text { hypersensitive troponin l; hsCRP, high-sensitivity C-reactive protein; DD, D-Dimer; Lymph, lymphocyte; } \\
\text { Neutrop, Neutrophil; CRP, C-reactive protein; PCT, procalcitonin; ALT, alanine transaminase; CysC, } \\
\text { Cystatin C. }\end{array}$} \\
\hline
\end{tabular}

The total population was divided into 2 groups by T2DM or not. Compared with non-T2DM patients, the value of age, hospital stay, the percentage of heart failure, severe inflammatory, liver injury in the hospital are higher in T2DM patients; The difference between the number of males and severe pneumonia are not significant. Refer to blood index, the value of CysC, IgBNP, lymphocyte, neutrophile, NLR, ALT, AST, blood glucose (Glu), alkaline phosphatase (ALP), choline kinase (CK), high-sensitivity C-reactive protein (hsCRP), D-Dimer on the baseline, the peak of NLR, CRP (C-reactive protein) are higher in T2DM group. Other than, the value of procalcitonin (PCT), albumin (ALB), creatinine, cardiac Troponin I (cTnl), creatine kinase isoenzymes (CK-MB) on the baseline, the peak of lymphocyte, neutrophile, PCT, ALT, AST, ALB, creatinine, ALP, CK, CK-MB, cTnl, CysC, hs-CRP, D-Dimer are not different between those 2 groups. It was worth mentioned mentioning that T2DM contributed to all-cause death. (Table 1).

\section{Characteristics of T2DM or not patients grouped by CysC level}

T2DM and non-T2DM patients were divided into 2 groups by CysC level, respectively. Among the T2DM patients, the CysC high group has a higher percentage of severe pneumonia, heart failure, death, a higher value of age than the CysC low group. The difference between males, severe inflammatory, and hospital stay are not significant. Refer to blood index, the value of CysC, IgBNP, PCT, ALB, Glu, cTnl, hs-CRP, DDimer on the baseline, the peak of ALB is higher in T2DM group. The value of lymphocyte, neutrophile, 
ALT, AST, Cre, CK, CK-MB on the baseline, the peak of lymphocyte, neutrophile, NLR, CRP, PCT, ALT, AST, Cre, CK, CK-MB, c Tnl, CysC, hs-CRP, D-Dimer is not different between those 2 groups.(Table 2) 
Table 2

characteristics of patients with or without DM classified by cystatin-C level

\begin{tabular}{|c|c|c|c|c|}
\hline Variables & Total & Cysc low & CysC high & $\mathbf{p}$ \\
\hline T2DM & $N=675$ & $N=344$ & $\mathrm{~N}=331$ & \\
\hline Age, y & $61(47.5,68)$ & $56(41,65)$ & $63(55,71)$ & $\begin{array}{l}< \\
0.001\end{array}$ \\
\hline Males, n (\%) & $328(49)$ & $165(48)$ & $163(49)$ & 0.798 \\
\hline $\begin{array}{l}\text { Severe pneumonia, } \mathrm{n} \\
(\%)\end{array}$ & $69(10.2)$ & $14(4.1)$ & $55(16.6)$ & $\begin{array}{l}<.001 \\
0.001\end{array}$ \\
\hline CysC baseline, mg/L & $0.9(0.74,1.12)$ & $0.84(0.71,1)$ & $0.99(0.78,1.18)$ & $\begin{array}{l}< \\
0.001\end{array}$ \\
\hline LgBNP baseline, $\mathrm{pg} / \mathrm{mL}$ & $1.21(0.38,1.66)$ & $1.09(0,1.57)$ & $1.32(0.65,1.75)$ & $\begin{array}{l}<.001 \\
0.001\end{array}$ \\
\hline Lymph baseline, $10^{9} / \mathrm{L}$ & $1.1(0.8,1.57)$ & $1.16(0.8,1.63)$ & $1.05(0.79,1.52)$ & 0.057 \\
\hline Neutrop baseline, $10^{9} / \mathrm{L}$ & $3.1(2.25,4.41)$ & $3.05(2.24,4.32)$ & $3.14(2.33,4.62)$ & 0.219 \\
\hline NLR baseline & $2.75(1.77,4.27)$ & $2.49(1.66,3.63)$ & $3.11(1.93,5.39)$ & 0.002 \\
\hline PCT baseline, ug/L & $0.03(0.03,0.05)$ & $0.03(0.03,0.03)$ & $0.03(0.03,0.05)$ & 0.048 \\
\hline ALT baseline, units/L & $26(16,39)$ & $26(17,37)$ & $26(16,39)$ & 0.838 \\
\hline AST baseline, units/L & $27(21,39)$ & $27(20,39)$ & $28(21,38)$ & 0.566 \\
\hline ALB baseline, $\mathrm{g} / \mathrm{L}$ & $37.38 \pm 5.02$ & $38.25 \pm 4.89$ & $36.48 \pm 5.01$ & $\dot{0.001}$ \\
\hline Glu baseline, $\mathrm{mmol} / \mathrm{L}$ & $5.68(4.86,7.2)$ & $5.51(4.74,7.19)$ & $5.87(4.98,7.23)$ & 0.034 \\
\hline Cre baseline, umol/L & $64.7(52.42,78.05)$ & $\begin{array}{l}63.5(52.15 \\
76.07)\end{array}$ & $\begin{array}{l}65.85(53.48, \\
81.62)\end{array}$ & 0.146 \\
\hline ALP baseline, units/L & $59(48,73)$ & $58(47.25,72)$ & $59(49,73)$ & 0.165 \\
\hline CK baseline, units/L & $71(46,124.25)$ & $70(47,119)$ & $72(46,130.5)$ & 0.716 \\
\hline CK-MB_baseline, $\mathrm{ng} / \mathrm{mL}$ & $10(8,14)$ & $10(7,14)$ & $11(8,14)$ & 0.069 \\
\hline cTnl baseline, ug/L & $0(0,0.01)$ & $0(0,0.01)$ & $0(0,0.01)$ & $\begin{array}{l}< \\
0.001\end{array}$ \\
\hline
\end{tabular}

Data are reported as mean $\pm S D$, median (IQR) or number and percentage. T2DM, Type 2 diabetes mellitus; BNP, Brain Natriuretic Peptide; NLR, Neutrophil-to-Lymphocyte Ratio; PCT, procalcitonin; ALT, Alanine aminotransferase; AST, Aspartate aminotransferase; ALB, albumin; Glu, glucose; Cre, creatinine; ALP, alkaline phosphatase; CK, choline kinase; CK-MB, creatine kinase isoenzymes; cTnl, hypersensitive troponin I; hsCRP, high-sensitivity C-reactive protein; DD, D-Dimer; Lymph, lymphocyte; Neutrop, Neutrophil; CRP, C-reactive protein; PCT, procalcitonin; ALT, alanine transaminase; CysC, Cystatin C. 


\begin{tabular}{|c|c|c|c|c|}
\hline Variables & Total & CysC low & CysC high & p \\
\hline hsCRP baseline, mg/L & $20(2.89,20)$ & $20(1.9,20)$ & $20(4.03,20)$ & 0.015 \\
\hline DD baseline, mg/L & $0.57(0.33,1.1)$ & $0.51(0.29,0.84)$ & $0.66(0.37,1.49)$ & $\begin{array}{l}< \\
0.001\end{array}$ \\
\hline Peak Lymph, $10^{9} / \mathrm{L}$ & $1.54(1.25,1.93)$ & $1.52(1.27,1.88)$ & $1.55(1.21,1.94)$ & 0.988 \\
\hline Peak Neutrop, $10^{9} / \mathrm{L}$ & $4.76(3.19,7.88)$ & $5.03(3.14,7.72)$ & $4.59(3.21,8.51)$ & 0.216 \\
\hline Peak NLR & $1.00(0.87,2.51)$ & $1.00(1.00,2.31)$ & $1.00(0.57,2.74)$ & 0.985 \\
\hline Peak CRP, mg/L & $\begin{array}{l}56.58(10.96 \\
150.96)\end{array}$ & $\begin{array}{l}52.42(7.98 \\
156.88)\end{array}$ & $\begin{array}{l}65.06(13.83 \\
138.53)\end{array}$ & 0.336 \\
\hline Peak PCT, ug/L & $0.48(0.15,2.58)$ & $0.54(0.18,2.72)$ & $0.36(0.12,2.58)$ & 0.372 \\
\hline Peak ALT, units/L & $48(28,91)$ & $48(31,107)$ & $48(25,84.5)$ & 0.204 \\
\hline Peak AST, units/L & $39(25,68.75)$ & $37(25,70)$ & $40(25.75,66)$ & 0.568 \\
\hline Peak ALB, g/L & $38.69 \pm 3.93$ & $39.42 \pm 3.91$ & $38.09 \pm 3.85$ & 0.011 \\
\hline Peak Cre, umol/L & $69(55.65,94.85)$ & $66.8(55,89.38)$ & $72.1(56,98.2)$ & 0.428 \\
\hline Peak ALP, units/L & $73(59,91)$ & $70(55.25,95.25)$ & $74(61.5,89.5)$ & 0.301 \\
\hline Peak CK, units/L & $85(53.75,246.5)$ & $73(51,244)$ & $108(54,263.5)$ & 0.917 \\
\hline Peak CK-MB, ng/mL & $14(10,26.25)$ & $14.5(9,27.25)$ & $13.5(11,24.5)$ & 0.989 \\
\hline Peak cTnl, ug/L & $0.09(0.02,0.42)$ & $0.1(0.01,0.42)$ & $0.08(0.02,0.48)$ & 0.86 \\
\hline Peak CysC, mg/L & $1.17(0.99,1.51)$ & $1.12(0.97,1.4)$ & $1.21(1.02,1.64)$ & 0.052 \\
\hline Peak hs-CRP, mg/L & $20(2.35,27)$ & $8.02(1.52,27.85)$ & $20(4.19,20)$ & 0.346 \\
\hline Peak DD, mg/L & $3.43(0.98,5.88)$ & $3.96(0.98,7.24)$ & $2.88(1.01,5.64)$ & 0.292 \\
\hline Hospital stays (days) & $14(11,17)$ & $14(11,17)$ & $14(10.5,18)$ & 0.842 \\
\hline Liver_injury, n (\%) & & & & 0.699 \\
\hline 0 (no) & $589(87)$ & 298 (87) & $291(88)$ & \\
\hline 1(yes) & $86(13)$ & $46(13)$ & $40(12)$ & \\
\hline Heart failure, n (\%) & & & & 0.033 \\
\hline \multicolumn{5}{|c|}{$\begin{array}{l}\text { Data are reported as mean } \pm \text { SD, median (IQR) or number and percentage. T2DM, Type } 2 \text { diabetes } \\
\text { mellitus; BNP, Brain Natriuretic Peptide; NLR, Neutrophil-to-Lymphocyte Ratio; PCT, procalcitonin; ALT, } \\
\text { Alanine aminotransferase; AST, Aspartate aminotransferase; ALB, albumin; Glu, glucose; Cre, } \\
\text { creatinine; ALP, alkaline phosphatase; CK, choline kinase; CK-MB, creatine kinase isoenzymes; cTnl, } \\
\text { hypersensitive troponin l; hsCRP, high-sensitivity C-reactive protein; DD, D-Dimer; Lymph, lymphocyte; } \\
\text { Neutrop, Neutrophil; CRP, C-reactive protein; PCT, procalcitonin; ALT, alanine transaminase; CysC, } \\
\text { Cystatin C. }\end{array}$} \\
\hline
\end{tabular}




\begin{tabular}{|c|c|c|c|c|}
\hline Variables & Total & Cysc low & CysC high & p \\
\hline 0 (no) & $655(97)$ & 339 (99) & $316(95)$ & \\
\hline 1 (yes) & $20(3)$ & $5(1)$ & $15(5)$ & \\
\hline $\begin{array}{l}\text { Severe inflammatory, } \mathrm{n} \\
(\%)\end{array}$ & & & & 0.952 \\
\hline 0 (no) & $641(95)$ & $326(95)$ & $315(95)$ & \\
\hline 1 (yes) & $34(5)$ & $18(5)$ & $16(5)$ & \\
\hline All-cause death, n (\%) & & & & 0.049 \\
\hline 0 (no) & $619(92)$ & $323(94)$ & $296(89)$ & \\
\hline 1 (yes) & $56(8)$ & $21(6)$ & $35(11)$ & \\
\hline Non-T2DM & $N=602$ & $N=301$ & $N=301$ & \\
\hline Age & $66(55,72)$ & $60(48,67)$ & $70(62,77)$ & $<.001$ \\
\hline Males, n (\%) & $281(47)$ & $125(42)$ & $156(52)$ & \\
\hline $\begin{array}{l}\text { Severe pneumonia, } \mathrm{n} \\
(\%)\end{array}$ & $51(8.5)$ & $18(6.0)$ & $33(11.0)$ & 0.108 \\
\hline CysC baseline, mg/L & $0.96(0.79,1.19)$ & $0.9(0.76,1.06)$ & $1.02(0.84,1.28)$ & $<.001$ \\
\hline LgBNP baseline, $\mathrm{pg} / \mathrm{mL}$ & $1.4(0.77,1.8)$ & $1.23(0.08,1.68)$ & $1.5(0.98,1.94)$ & $\stackrel{<}{0.001}$ \\
\hline Lymph baseline, $10^{9} / \mathrm{L}$ & $1.19(0.86,1.62)$ & $1.28(0.88,1.68)$ & $1.11(0.82,1.54)$ & 0.008 \\
\hline Neutrop baseline, $10^{9} / \mathrm{L}$ & $3.37(2.54,4.64)$ & $3.29(2.5,4.49)$ & $3.46(2.57,4.81)$ & 0.287 \\
\hline NLR baseline & $2.50(1.70,3.68)$ & $2.40(1.69,3.37)$ & $2.62(1.69,4.49)$ & 0.206 \\
\hline PCT baseline, ug/L & $0.03(0.03,0.04)$ & $0.03(0.03,0.03)$ & $0.03(0.03,0.06)$ & 0.163 \\
\hline ALT baseline, units/L & $22(15,35)$ & $20(14,32)$ & $24(16,40)$ & 0.002 \\
\hline AST baseline, units/L & $25(19,34)$ & $23(18,30)$ & $28(21,37)$ & 0.000 \\
\hline ALB baseline, $\mathrm{g} / \mathrm{L}$ & $37.47 \pm 5.13$ & $38.33 \pm 5.07$ & $36.6 \pm 5.06$ & $<.001$ \\
\hline \multicolumn{5}{|c|}{$\begin{array}{l}\text { Data are reported as mean } \pm \text { SD, median (IQR) or number and percentage. T2DM, Type } 2 \text { diabetes } \\
\text { mellitus; BNP, Brain Natriuretic Peptide; NLR, Neutrophil-to-Lymphocyte Ratio; PCT, procalcitonin; ALT, } \\
\text { Alanine aminotransferase; AST, Aspartate aminotransferase; ALB, albumin; Glu, glucose; Cre, } \\
\text { creatinine; ALP, alkaline phosphatase; CK, choline kinase; CK-MB, creatine kinase isoenzymes; cTnl, } \\
\text { hypersensitive troponin l; hsCRP, high-sensitivity C-reactive protein; DD, D-Dimer; Lymph, lymphocyte; } \\
\text { Neutrop, Neutrophil; CRP, C-reactive protein; PCT, procalcitonin; ALT, alanine transaminase; CysC, } \\
\text { Cystatin C. }\end{array}$} \\
\hline
\end{tabular}




\begin{tabular}{|c|c|c|c|c|}
\hline Variables & Total & CysC low & CysC high & p \\
\hline Glu_baseline, $\mathrm{mmol} / \mathrm{L}$ & $5.25(4.65,6.58)$ & $5.2(4.63,6.38)$ & $5.33(4.68,6.69)$ & 0.135 \\
\hline Cre baseline, umol/L & $60.7(50,78.4)$ & $\begin{array}{l}57.65(48.12, \\
74.4)\end{array}$ & $64.4(53.2,83.15)$ & <. 001 \\
\hline ALP baseline, units/L & $63(51,78)$ & $60(50.75,77.25)$ & $65(53,79)$ & 0.101 \\
\hline CK baseline, units/L & $65(44,107)$ & $60(43,96)$ & $67.5(48,116)$ & 0.023 \\
\hline CK-MB_baseline, $\mathrm{ng} / \mathrm{mL}$ & $10(7,13)$ & $9(7,12)$ & $10(7,14)$ & 0.01 \\
\hline cTnl baseline, ug/L & $0(0,0.01)$ & $0(0,0.01)$ & $0.01(0,0.01)$ & $\begin{array}{l}< \\
0.001\end{array}$ \\
\hline hsCRP baseline, mg/L & $7.1(1.9,20)$ & $4.9(1.51,20)$ & $9.94(2.45,20)$ & $\begin{array}{l}<.001 \\
0.001\end{array}$ \\
\hline DD baseline, mg/L & $0.69(0.39,2.15)$ & $0.6(0.35,1.18)$ & $0.92(0.45,3.12)$ & $\begin{array}{l}< \\
0.001\end{array}$ \\
\hline Peak Lymph, $10^{9} / \mathrm{L}$ & $1.47(1.11,1.91)$ & $1.55(1.16,2.03)$ & $1.43(1.08,1.85)$ & 0.326 \\
\hline Peak Neutrop, $10^{9} / \mathrm{L}$ & $4.72(3.40,7.01)$ & $4.68(3.26,6.45)$ & $4.88(3.53,7.88)$ & 0.800 \\
\hline Peak NLR & $2.06(0.32,5.77)$ & $1.83(0.44,7.57)$ & $2.45(1.00,5.09)$ & 0.403 \\
\hline Peak CRP, mg/L & $15.48(3.56,71.52)$ & $9.37(3.04,40.81)$ & $25.89(5.03,78.56)$ & 0.058 \\
\hline Peak PCT, ug/L & $0.26(0.1,2.46)$ & $0.35(0.17,2.67)$ & $0.2(0.1,1.16)$ & 0.396 \\
\hline Peak ALT, units/L & $42(24,69.25)$ & $36(23,68)$ & $48(27.5,74)$ & 0.073 \\
\hline Peak AST, units/L & $33(24,56)$ & $29.5(21.0,53.0)$ & $38.0(28.0,68.0)$ & 0.004 \\
\hline Peak ALB, g/L & $38.59 \pm 4.19$ & $39.1 \pm 4.28$ & $38.16 \pm 4.08$ & 0.107 \\
\hline Peak Cre, umol/L & $65.2(53.65,80.3)$ & $61(50.55,73.15)$ & $69.9(56.4,83.4)$ & 0.01 \\
\hline Peak ALP, units/L & $76(63,94)$ & $76.5(63.25,91)$ & $75(61,99)$ & 0.603 \\
\hline Peak CK, units/L & $63(44.5,173)$ & $53(43.75,75.5)$ & $75(47,218)$ & 0.128 \\
\hline Peak CK-MB, ng/mL & $13(10,21)$ & $11(9,18.75)$ & $14(10.5,22)$ & 0.025 \\
\hline Peak cTnl, ug/L & $0.05(0.02,0.7)$ & $0.02(0.01,0.11)$ & $0.09(0.03,0.95)$ & 0.132 \\
\hline
\end{tabular}

Data are reported as mean $\pm S D$, median (IQR) or number and percentage. T2DM, Type 2 diabetes mellitus; BNP, Brain Natriuretic Peptide; NLR, Neutrophil-to-Lymphocyte Ratio; PCT, procalcitonin; ALT, Alanine aminotransferase; AST, Aspartate aminotransferase; ALB, albumin; Glu, glucose; Cre, creatinine; ALP, alkaline phosphatase; CK, choline kinase; CK-MB, creatine kinase isoenzymes; cTnl, hypersensitive troponin I; hsCRP, high-sensitivity C-reactive protein; DD, D-Dimer; Lymph, lymphocyte; Neutrop, Neutrophil; CRP, C-reactive protein; PCT, procalcitonin; ALT, alanine transaminase; CysC, Cystatin C. 


\begin{tabular}{|c|c|c|c|c|}
\hline Variables & Total & CysC low & CysC high & p \\
\hline Peak CysC, mg/L & $1.21(0.98,1.57)$ & $1.09(0.91,1.36)$ & $1.3(1.04,1.74)$ & $\begin{array}{l}< \\
0.001\end{array}$ \\
\hline Peak hs-CRP, mg/L & $6.28(2.02,20)$ & $2.42(1.7,6.28)$ & $20(3.73,20)$ & 0.005 \\
\hline Peak DD, mg/L & $2.96(0.9,6.32)$ & $1.65(0.77,4.94)$ & $4.4(1.35,7.15)$ & 0.001 \\
\hline Hospital stays (days) & $16(14,17)$ & $16(14,17)$ & $16(13,18)$ & 0.317 \\
\hline Liver injury, n (\%) & & & & 0.761 \\
\hline 0 & $555(92)$ & 279 (93) & $276(92)$ & \\
\hline 1 & $47(8)$ & $22(7)$ & $25(8)$ & \\
\hline Heart failure, n (\%) & & & & 0.004 \\
\hline 0 & $577(96)$ & $296(98)$ & $281(93)$ & \\
\hline 1 & $25(4)$ & $5(2)$ & $20(7)$ & \\
\hline $\begin{array}{l}\text { Severe inflammatory, } \mathrm{n} \\
(\%)\end{array}$ & & & & 0.811 \\
\hline 0 & $521(87)$ & 262 (87) & $259(86)$ & \\
\hline 1 & $81(13)$ & $39(13)$ & $42(14)$ & \\
\hline All-cause death, $\mathrm{n}(\%)$ & & & & 1 \\
\hline 0 & $602(100)$ & $301(100)$ & $301(100)$ & \\
\hline \multicolumn{5}{|c|}{$\begin{array}{l}\text { Data are reported as mean } \pm \text { SD, median (IQR) or number and percentage. T2DM, Type } 2 \text { diabetes } \\
\text { mellitus; BNP, Brain Natriuretic Peptide; NLR, Neutrophil-to-Lymphocyte Ratio; PCT, procalcitonin; ALT, } \\
\text { Alanine aminotransferase; AST, Aspartate aminotransferase; ALB, albumin; Glu, glucose; Cre, } \\
\text { creatinine; ALP, alkaline phosphatase; CK, choline kinase; CK-MB, creatine kinase isoenzymes; cTnl, } \\
\text { hypersensitive troponin I; hsCRP, high-sensitivity C-reactive protein; DD, D-Dimer; Lymph, lymphocyte; } \\
\text { Neutrop, Neutrophil; CRP, C-reactive protein; PCT, procalcitonin; ALT, alanine transaminase; CysC, } \\
\text { Cystatin C. }\end{array}$} \\
\hline
\end{tabular}

As for the non-T2DM patients, the CysC high group has a higher percentage of males, heart failure, higher value of age than the CysC low group. The difference in severe pneumonia and hospital stay, liver injury, severe inflammatory are not significant. Refer to blood index, the value of CysC, IgBNP, lymphocyte, ALT, AST, ALB, Glu, Cre, cTnl, hs-CRP, D-Dimer on the baseline, the peak of cre, CysC, and hs-CRP are higher in the T2DM group. The value of neutrophile, PCT, CK, CK-MB on the baseline, the peak of lymphocyte, neutrophile, CRP, PCT, ALT, AST, Cre, CK, CK-MB, c Tnl, D-Dimer are not different between those 2 groups. (Table 2) 
T2DM and non-T2DM patients were divided into 2 groups by CysC rangeability, respectively. Among the T2DM patients, the CysC rangeability $>0$ group has a higher percentage of severe pneumonia, severe inflammatory, liver injury, a higher value of age than the $\mathrm{CysC}$ rangeability $\leq 0$ group. The difference between the males, hospital stay, heart failure, and death is not significant. Refer to blood index, the value of CysC, IgBNP, lymphocyte, ALT, AST, ALB, CTnl, hs-CRP, D-Dimer on the baseline, the peak of CRP are higher in CysC rangeability>0 groups. The value of neutrophile, creatinine (Cre), PCT, ALP, CK, CK-MB on the baseline, the peak of ALB, lymphocyte, neutrophile, NLR, PCT, ALT, AST, Cre, ALP, CK, CK-MB, C Tnl, CysC, hs-CRP, D-Dimer are not different between those 2 groups. (Table 3 ) 
Table 3

characteristics of patients with or without T2DM classified by CysC rangeability

\begin{tabular}{|c|c|c|c|c|}
\hline Variables & Total & $\begin{array}{l}\text { CysC rangeability } \\
\leq 0\end{array}$ & $\begin{array}{l}\text { CysC } \\
\text { rangeability >0 }\end{array}$ & $\mathbf{p}$ \\
\hline T2DM & $N=675$ & $N=407$ & $N=268$ & \\
\hline Age, years & $61(47.5,68)$ & $56(41,65)$ & $63(55,71)$ & $<.001$ \\
\hline Males, n (\%) & $328(49)$ & $193(47.4)$ & $135(50.4)$ & \\
\hline $\begin{array}{l}\text { Severe pneumonia, } \mathrm{n} \\
(\%)\end{array}$ & $69(10.2)$ & $12(2.9)$ & $57(21.3)$ & $\begin{array}{l}< \\
0.001\end{array}$ \\
\hline CysC baseline, mg/L & $0.96(0.79,1.19)$ & $0.9(0.76,1.06)$ & $1.02(0.84,1.28)$ & <. \\
\hline LgBNP baseline, $\mathrm{pg} / \mathrm{mL}$ & $1.4(0.77,1.8)$ & $1.23(0.08,1.68)$ & $1.5(0.98,1.94)$ & $<.001$ \\
\hline Lymph_baseline, $10^{9} / \mathrm{L}$ & $1.19(0.86,1.62)$ & $1.28(0.88,1.68)$ & $1.11(0.82,1.54)$ & 0.008 \\
\hline $\begin{array}{l}\text { Neutrop_baseline, } \\
10^{9} / \mathrm{L}\end{array}$ & $3.37(2.54,4.64)$ & $3.29(2.5,4.49)$ & $3.46(2.57,4.81)$ & 0.287 \\
\hline NLR baseline & $2.50(1.70,3.68)$ & $2.24(1.62,3.09)$ & $3.34(2.42,5.93)$ & 0.206 \\
\hline PCT baseline, ug/L & $0.25(0.25,0.25)$ & $0.25(0.25,0.25)$ & $0.25(0.25,0.58)$ & 0.163 \\
\hline ALT baseline, units/L & $26(16,39)$ & $24(16,38)$ & $26(18,40)$ & 0.002 \\
\hline AST baseline, units/L & $27(21,38)$ & $26(20,37)$ & $30(21,42)$ & 0.000 \\
\hline ALB baseline, $\mathrm{g} / \mathrm{L}$ & $37.15 \pm 4.33$ & $37.64 \pm 4.21$ & $36.25 \pm 4.76$ & <. \\
\hline Glu baseline, $\mathrm{mmol} / \mathrm{L}$ & $5.67(4.84,7.19)$ & $5.62(4.87,7.13)$ & $5.76(4.80,7.33)$ & 0.135 \\
\hline Cre baseline, umol/L & $\begin{array}{l}65.10(52.40 \\
78.70)\end{array}$ & $\begin{array}{l}64.20(51.60 \\
78.20)\end{array}$ & $65.5(54.40,80.80)$ & $\begin{array}{l}<.001 \\
\end{array}$ \\
\hline ALP baseline, units/L & $\begin{array}{l}59.00(48.00 \\
73.00)\end{array}$ & $\begin{array}{l}57.00(47.00 \\
72.00)\end{array}$ & $\begin{array}{l}61.00(49.00 \\
74.00)\end{array}$ & 0.101 \\
\hline CK baseline, units/L & $\begin{array}{l}72.00(46.00 \\
129.00)\end{array}$ & $\begin{array}{l}68.00(45.00 \\
123.00)\end{array}$ & $\begin{array}{l}76.50(48.25 \\
136.75)\end{array}$ & 0.023 \\
\hline CK-MB_baseline, $\mathrm{ng} / \mathrm{mL}$ & $10(8,14)$ & $10(8,13)$ & $11(7,14)$ & 0.01 \\
\hline \multicolumn{5}{|c|}{$\begin{array}{l}\text { Data are reported as mean } \pm \text { SD, median (IQR) or number and percentage. T2DM, Type } 2 \text { diabetes } \\
\text { mellitus; BNP, Brain Natriuretic Peptide; NLR, Neutrophil-to-Lymphocyte Ratio; PCT, procalcitonin; ALT, } \\
\text { Alanine aminotransferase; AST, Aspartate aminotransferase; ALB, albumin; Glu, glucose; Cre, } \\
\text { creatinine; ALP, alkaline phosphatase; CK, choline kinase; CK-MB, creatine kinase isoenzymes; cTnl, } \\
\text { hypersensitive troponin l; hsCRP, high-sensitivity C-reactive protein; DD, D-Dimer; Lymph, lymphocyte; } \\
\text { Neutrop, Neutrophil; CRP, C-reactive protein; PCT, procalcitonin; ALT, alanine transaminase; CysC, } \\
\text { Cystatin C. }\end{array}$} \\
\hline
\end{tabular}




\begin{tabular}{|c|c|c|c|c|}
\hline Variables & Total & $\begin{array}{l}\text { CysC rangeability } \\
\leq 0\end{array}$ & $\begin{array}{l}\text { CysC } \\
\text { rangeability>0 }\end{array}$ & p \\
\hline cTnl baseline, ug/L & $0(0,0.01)$ & $0(0,0.01)$ & $0.01(0,0.01)$ & $<.001$ \\
\hline hsCRP baseline, mg/L & $20.0(2.8,20.0)$ & $20.0(2.30,20.0)$ & $20.0(3.14,20.0)$ & $<.001$ \\
\hline DD baseline, mg/L & $0.69(0.39,2.15)$ & $0.6(0.35,1.18)$ & $0.92(0.45,3.12)$ & $\begin{array}{l}< \\
0.001\end{array}$ \\
\hline Peak Lymph, $10^{9} / \mathrm{L}$ & $1.53(1.25,1.93)$ & $1.54(1.23,1.93)$ & $1.53(1.25,1.93)$ & 0.245 \\
\hline Peak Neutrop, $10^{9} / \mathrm{L}$ & $4.76(3.19,7.88)$ & $4.81(3.16,7.63)$ & $4.66(3.42,9.38)$ & 0.803 \\
\hline Peak NLR & $1.00(0.87,2.51)$ & $1.00(1.00,2.40)$ & $1.00(1.00,2.92)$ & 0.611 \\
\hline Peak CRP, mg/L & $\begin{array}{l}56.58(10.38 \\
152.32)\end{array}$ & $\begin{array}{l}58.76(12.75 \\
149.93)\end{array}$ & $\begin{array}{l}52.43(8.65 \\
138.53)\end{array}$ & 0.027 \\
\hline Peak PCT, ug/L & $0.48(0.15,2.69)$ & $0.37(0.10,4.34)$ & $0.51(0.18,2.43)$ & 0.901 \\
\hline Peak ALT, units/L & $49.5(28.25,96.75)$ & $\begin{array}{l}43.00(28.00 \\
84.50)\end{array}$ & $\begin{array}{l}54.00(29.00 \\
107.00)\end{array}$ & 0.058 \\
\hline Peak AST, units/L & $39(25,68.75)$ & $35(24,59)$ & $44(27,77.5)$ & 0.066 \\
\hline Peak ALB, g/L & $38.69 \pm 3.93$ & $38.60 \pm 3.79$ & $38.25 \pm 4.04$ & 0.691 \\
\hline Peak Cre, umol/L & $69(55.65,94.85)$ & $67.8(54.1,100.1)$ & $74.5(59.6,105.0)$ & 0.280 \\
\hline Peak ALP, units/L & $73(59,91)$ & $\begin{array}{l}70.50(59.00 \\
85.25)\end{array}$ & $\begin{array}{l}75.00(59.00 \\
101.50)\end{array}$ & 0.021 \\
\hline Peak CK, units/L & $85(53.75,246.5)$ & $85(45.00,398.00)$ & $115(63,459)$ & 0.522 \\
\hline Peak CK-MB, ng/mL & $14.0(10,26.25)$ & $14.0(9,27.25)$ & $15.0(10.0,31.0)$ & 0.338 \\
\hline Peak cTnl, ug/L & $0.09(0.02,0.42)$ & $0.1(0.98,1.52)$ & $1.20(1.00,1.50)$ & 0.909 \\
\hline Peak CysC, mg/L & $1.17(0.99,1.51)$ & $1.15(0.97,1.4)$ & $1.21(1.02,1.64)$ & 0.717 \\
\hline Peak hs-CRP, mg/L & $20(2.35,27)$ & $8.02(1.52,27.85)$ & $20(4.19,20)$ & 0.297 \\
\hline Peak DD, mg/L & $3.43(0.98,5.88)$ & $3.11(0.97,5.48)$ & $3.70(1.04,7.21)$ & 0.936 \\
\hline Hospital stays (days) & $14(11,17)$ & $14(10,17)$ & $14(11,18)$ & 0.071 \\
\hline
\end{tabular}

Data are reported as mean $\pm S D$, median (IQR) or number and percentage. T2DM, Type 2 diabetes mellitus; BNP, Brain Natriuretic Peptide; NLR, Neutrophil-to-Lymphocyte Ratio; PCT, procalcitonin; ALT, Alanine aminotransferase; AST, Aspartate aminotransferase; ALB, albumin; Glu, glucose; Cre, creatinine; ALP, alkaline phosphatase; CK, choline kinase; CK-MB, creatine kinase isoenzymes; CTnl, hypersensitive troponin I; hsCRP, high-sensitivity C-reactive protein; DD, D-Dimer; Lymph, lymphocyte; Neutrop, Neutrophil; CRP, C-reactive protein; PCT, procalcitonin; ALT, alanine transaminase; CysC, Cystatin C. 


\begin{tabular}{|c|c|c|c|c|}
\hline Variables & Total & $\begin{array}{l}\text { CysC rangeability } \\
\leq 0\end{array}$ & $\begin{array}{l}\text { CysC } \\
\text { rangeability }>0\end{array}$ & p \\
\hline Liver injury, n (\%) & & & & 0.020 \\
\hline 0 (no) & $589(87)$ & 365 (89.7) & $224(83.6)$ & \\
\hline 1 (yes) & $86(13)$ & $42(10.3)$ & $44(16.4)$ & \\
\hline Heart failure, n (\%) & & & & 0.632 \\
\hline 0 (no) & $655(97)$ & $396(97.3)$ & $259(96.6)$ & \\
\hline 1 (yes) & $20(3)$ & $11(2.7)$ & $9(3.4)$ & \\
\hline $\begin{array}{l}\text { Severe inflammation, } \mathrm{n} \\
(\%)\end{array}$ & & & & 0.048 \\
\hline 0 (no) & $641(95)$ & $392(96.3)$ & 249 (92.9) & \\
\hline 1 (yes) & $34(5)$ & $15(3.7)$ & $19(7.1)$ & \\
\hline All-cause Death, n (\%) & & & & 0.283 \\
\hline 0 (no) & $619(92)$ & $377(92.6)$ & $242(90.3)$ & \\
\hline 1 (yes) & $56(8)$ & $30(7.4)$ & $26(9.7)$ & \\
\hline Non-T2DM & $N=602$ & $N=386$ & $N=216$ & \\
\hline Age & $66(55,72)$ & $60(48,67)$ & $70(62,77)$ & $\begin{array}{l}< \\
0.001\end{array}$ \\
\hline Males, n (\%) & $281(47)$ & $176(45.6)$ & $105(48.6)$ & 0.477 \\
\hline $\begin{array}{l}\text { Severe pneumonia, } \mathrm{n} \\
(\%)\end{array}$ & $51(8.5)$ & $15(3.9)$ & $36(16.7)$ & $\dot{0.001}$ \\
\hline CysC baseline, mg/L & $0.95(0.79,1.19)$ & $0.94(0.78,1.18)$ & $0.96(0.81,1.22)$ & $\begin{array}{l}< \\
0.001\end{array}$ \\
\hline LgBNP baseline, $\mathrm{pg} / \mathrm{mL}$ & $1.39(0.77,1.80)$ & $1.33(0.17,1.78)$ & $1.48(0.98,1.88)$ & $<0.001$ \\
\hline Lymph baseline, $10^{9} / \mathrm{L}$ & $1.19(0.85,1.62)$ & $1.20(0.86,1.64)$ & $1.17(0.82,1.58)$ & 0.008 \\
\hline Neutrop baseline, $10^{9} / \mathrm{L}$ & $3.37(2.51,4.64)$ & $3.31(2.45,4.51)$ & $3.55(2.66,4.83)$ & 0.287 \\
\hline NLR baseline & $2.75(1.77,4.27)$ & $2.35(1.59,3.52)$ & $3.30(2.19,5.99)$ & 0.206 \\
\hline \multicolumn{5}{|c|}{$\begin{array}{l}\text { Data are reported as mean } \pm \text { SD, median (IQR) or number and percentage. T2DM, Type } 2 \text { diabetes } \\
\text { mellitus; BNP, Brain Natriuretic Peptide; NLR, Neutrophil-to-Lymphocyte Ratio; PCT, procalcitonin; ALT, } \\
\text { Alanine aminotransferase; AST, Aspartate aminotransferase; ALB, albumin; Glu, glucose; Cre, } \\
\text { creatinine; ALP, alkaline phosphatase; CK, choline kinase; CK-MB, creatine kinase isoenzymes; cTnl, } \\
\text { hypersensitive troponin l; hsCRP, high-sensitivity C-reactive protein; DD, D-Dimer; Lymph, lymphocyte; } \\
\text { Neutrop, Neutrophil; CRP, C-reactive protein; PCT, procalcitonin; ALT, alanine transaminase; CysC, } \\
\text { Cystatin C. }\end{array}$} \\
\hline
\end{tabular}




\begin{tabular}{|c|c|c|c|c|}
\hline Variables & Total & $\begin{array}{l}\text { CysC rangeability } \\
\leq 0\end{array}$ & $\begin{array}{l}\text { CysC } \\
\text { rangeability>0 }\end{array}$ & p \\
\hline PCT baseline, ug/L & $0.25(0.25,0.25)$ & $0.25(0.25,0.25)$ & $0.25(0.25,0.58)$ & 0.163 \\
\hline ALT baseline, units/L & $22(14.75,35.00)$ & $\begin{array}{l}22.00(14.00 \\
35.00)\end{array}$ & $\begin{array}{l}27.00(20.00 \\
35.75)\end{array}$ & 0.002 \\
\hline AST baseline, units/L & $25(19,34)$ & $24(19,33.25)$ & $\begin{array}{l}27.00(20.00 \\
35.75)\end{array}$ & 0.000 \\
\hline ALB baseline, $\mathrm{g} / \mathrm{L}$ & $37.40 \pm 3.37$ & $37.58 \pm 3.82$ & $37.08 \pm 3.97$ & $\begin{array}{l}< \\
0.001\end{array}$ \\
\hline Glu baseline, $\mathrm{mmol} / \mathrm{L}$ & $5.24(4.63,6.58)$ & $5.21(4.61,6.58)$ & $5.37(4.67,6.63)$ & 0.135 \\
\hline Cre baseline, umol/L & $\begin{array}{l}61.25(50.20, \\
80.23)\end{array}$ & $\begin{array}{l}\text { 60.45(50.15, } \\
78.18)\end{array}$ & $\begin{array}{l}62.95(50.32, \\
85.42)\end{array}$ & $\begin{array}{l}< \\
0.001\end{array}$ \\
\hline ALP baseline, units/L & $\begin{array}{l}63.00(51.00 \\
78.00)\end{array}$ & $\begin{array}{l}64.00(52.00 \\
81.00)\end{array}$ & $\begin{array}{l}59.00(48.00 \\
73.00)\end{array}$ & 0.101 \\
\hline CK baseline, units/L & $\begin{array}{l}65.00(44.00 \\
110.25)\end{array}$ & $\begin{array}{l}63.50(44.00 \\
105.50)\end{array}$ & $\begin{array}{l}68.00(43.25 \\
115.75)\end{array}$ & 0.023 \\
\hline CK-MB_baseline, $\mathrm{ng} / \mathrm{mL}$ & $10(7,13)$ & $9(7,14)$ & $10(7,13)$ & 0.01 \\
\hline cTnl baseline, ug/L & $0(0,0.01)$ & $0(0,0.01)$ & $0.01(0,0.01)$ & $\begin{array}{l}< \\
0.001\end{array}$ \\
\hline hsCRP baseline, mg/L & $7.04(1.88,20.00)$ & $5.13(1.60,20.00)$ & $10.1(2.81,20.0)$ & $\begin{array}{l}< \\
0.001\end{array}$ \\
\hline DD baseline, mg/L & $0.69(0.39,2.15)$ & $0.66(0.38,1.92)$ & $0.81(0.41,2.96)$ & 0.167 \\
\hline Peak Lymph, $10^{9} / \mathrm{L}$ & $1.47(1.11,1.91)$ & $1.44(1.10,1.88)$ & $1.58(1.11,1.97)$ & 0.309 \\
\hline Peak Neutrop, $10^{9} / \mathrm{L}$ & $4.72(3.40,7.01)$ & $4.60(3.41,6.37)$ & $4.85(3.36,7.98)$ & 0.698 \\
\hline Peak NLR & $2.06(0.32,5.77)$ & $1.74(1.00,5.09)$ & $2.54(0.52,8.24)$ & 0.052 \\
\hline Peak CRP, mg/L & $15.48(3.56,71.52)$ & $9.78(2.53,63.12)$ & $25.36(5.41,73.60)$ & 0.100 \\
\hline Peak PCT, ug/L & $0.26(0.1,2.46)$ & $0.23(0.10,0.75)$ & $0.80(0.1,4.15)$ & 0.490 \\
\hline Peak ALT, units/L & $42(24,69.25)$ & $38(22,65)$ & $50(27,77)$ & 0.031 \\
\hline Peak AST, units/L & $33(24,56)$ & $31.0(22.0,50.0)$ & $35.0(28.0,60.3)$ & 0.263 \\
\hline
\end{tabular}

Data are reported as mean $\pm S D$, median (IQR) or number and percentage. T2DM, Type 2 diabetes mellitus; BNP, Brain Natriuretic Peptide; NLR, Neutrophil-to-Lymphocyte Ratio; PCT, procalcitonin; ALT, Alanine aminotransferase; AST, Aspartate aminotransferase; ALB, albumin; Glu, glucose; Cre, creatinine; ALP, alkaline phosphatase; CK, choline kinase; CK-MB, creatine kinase isoenzymes; CTnl, hypersensitive troponin I; hsCRP, high-sensitivity C-reactive protein; DD, D-Dimer; Lymph, lymphocyte; Neutrop, Neutrophil; CRP, C-reactive protein; PCT, procalcitonin; ALT, alanine transaminase; CysC, Cystatin C. 


\begin{tabular}{|c|c|c|c|c|}
\hline Variables & Total & $\begin{array}{l}\text { CysC rangeability } \\
\leq 0\end{array}$ & $\begin{array}{l}\text { CysC } \\
\text { rangeability>0 }\end{array}$ & p \\
\hline Peak ALB, g/L & $38.59 \pm 4.19$ & $38.3 \pm 3.81$ & $38.5 \pm 4.63$ & 0.951 \\
\hline Peak Cre, umol/L & $65.2(53.65,80.3)$ & $\begin{array}{l}66.85(54.53 \\
85.28)\end{array}$ & $64.9(53.2,80.0)$ & 0.66 \\
\hline Peak ALP, units/L & $76(63,94)$ & $77.0(62.0,91.0)$ & $74.0(63.3,91.2)$ & 0.812 \\
\hline Peak CK, units/L & $63(44.5,173)$ & $\begin{array}{l}70.00(49.00 \\
244.00)\end{array}$ & $\begin{array}{l}71.00(42.00 \\
314.75)\end{array}$ & 0.899 \\
\hline Peak CK-MB, ng/mL & $13(10,21)$ & $13(10,21)$ & $14(10,21.25)$ & 0.587 \\
\hline Peak cTnl, ug/L & $0.05(0.02,0.7)$ & $0.03(0.01,0.09)$ & $0.12(0.02,1.90)$ & 0.167 \\
\hline Peak CysC, mg/L & $1.21(0.98,1.57)$ & $1.22(0.94,1.59)$ & $1.20(1.00,1.55)$ & 0.87 \\
\hline Peak hs-CRP, mg/L & $6.28(2.02,20)$ & $6.74(1.90,20.00)$ & $5.57(2.21,20.00)$ & 0.751 \\
\hline Peak DD, mg/L & $2.96(0.9,6.32)$ & $3.60(1.09,6.31)$ & $2.36(0.79,6.36)$ & 0.331 \\
\hline Hospital stays (days) & $16(14,17)$ & $16(9,17)$ & $16(14,18)$ & 0.004 \\
\hline Liver injury, n (\%) & & & & 0.104 \\
\hline 0 (no) & $555(92)$ & $194(89.8)$ & 361 (93.5) & \\
\hline 1 (yes) & $47(8)$ & $22(10.2)$ & $25(6.5)$ & \\
\hline Heart failure, n (\%) & & & & 0.400 \\
\hline 0 (no) & $577(96)$ & $205(94.9)$ & $372(96.4)$ & \\
\hline 1 (yes) & $25(4)$ & $11(5.1)$ & $14(3.6)$ & \\
\hline $\begin{array}{l}\text { Severe inflammation, } \mathrm{n} \\
(\%)\end{array}$ & & & & 0.084 \\
\hline 0 (no) & $521(87)$ & 341 (88.3) & $180(83.3)$ & \\
\hline 1 (yes) & $81(13)$ & $45(11.7)$ & $36(16.7)$ & \\
\hline All-cause death, $\mathrm{n}(\%)$ & & & & 1 \\
\hline 0 (no) & $602(100)$ & $301(100)$ & $301(100)$ & \\
\hline \multicolumn{5}{|c|}{$\begin{array}{l}\text { Data are reported as mean } \pm \text { SD, median (IQR) or number and percentage. T2DM, Type } 2 \text { diabetes } \\
\text { mellitus; BNP, Brain Natriuretic Peptide; NLR, Neutrophil-to-Lymphocyte Ratio; PCT, procalcitonin; ALT, } \\
\text { Alanine aminotransferase; AST, Aspartate aminotransferase; ALB, albumin; Glu, glucose; Cre, } \\
\text { creatinine; ALP, alkaline phosphatase; CK, choline kinase; CK-MB, creatine kinase isoenzymes; cTnl, } \\
\text { hypersensitive troponin I; hsCRP, high-sensitivity C-reactive protein; DD, D-Dimer; Lymph, lymphocyte; } \\
\text { Neutrop, Neutrophil; CRP, C-reactive protein; PCT, procalcitonin; ALT, alanine transaminase; CysC, } \\
\text { Cystatin C. }\end{array}$} \\
\hline
\end{tabular}


In the non-diabetic population, the $\mathrm{Cys} C$ rangeability $>0$ group has a higher percentage of severe pneumonia, higher value of age, hospital stay than CysC rangeability $\leq 0$. The difference between liver injury, severe inflammatory, heart failure is not significant. Refer to blood index, the value of CysC, IgBNP, lymphocyte, ALT, AST, ALB, creatinine, CK, CK-MB, cTnl, hs-CRP, D-Dimer on the baseline, the peak of cre and ALT is higher in CysC rangeability $>0$ group. The value of neutrophile, PCT, CK, CK-MB, glucose on the baseline, the peak of lymphocyte, neutrophile, CRP, PCT, ALT, CysC, and hs-CRP CK, CK-MB, cTnl, D-Dimer are not different between those 2 groups. (Table 3 )

\section{The association between CysC and Organ dysfunction, all-cause death classified by T2DM}

Multivariate logistic analysis for organ dysfunction and all-cause death showed that when CysC $\leq$ $0.93 \mathrm{mg} / \mathrm{dl}$ as a reference, CysC $>0.93 \mathrm{mg} / \mathrm{dl}$ were significantly associated with the risk of heart failure $(\mathrm{OR}=2.401,95 \% \mathrm{Cl}: 1.118-5.156)$ and all-cause death $(\mathrm{OR}=2.734,95 \% \mathrm{Cl}: 1.098-6.811)$, but the association did not go for severe inflammatory $(\mathrm{OR}=0.98,95 \% \mathrm{Cl}: 0.661-1.446)$ and liver injury $(\mathrm{OR}=$ 1.168, 95\% Cl: 0.789-1.729), (Fig. 1).

Furthermore, classified by T2DM status, using CysC $\leq 0.93 \mathrm{mg} / \mathrm{dl}$ as a reference standard, CysC > $0.93 \mathrm{mg} / \mathrm{dl}$ had an increased risk of heart failure $(\mathrm{OR}=2.962,95 \% \mathrm{Cl}: 1.006-8.724)$ and all-cause death $(\mathrm{OR}=5.585,95 \% \mathrm{Cl}: 2.328-13.397)$, but not for severe inflammatory (OR $=0.81,95 \% \mathrm{Cl}: 0.402-1.647)$ and liver injury ( $\mathrm{OR}=0.986,95 \% \mathrm{Cl}: 0.601-1.618)$ for T2DM patients. Whereas the relationship between CysC $>0.93 \mathrm{mg} / \mathrm{dl}$ and severe inflammatory, liver injury, or heart failure was not significant in the nonT2DM group (Fig. 1).

When goes for sex subgroup of T2DM patients, adjusted by age, severe pneumonia, serum albumin, blood glucose, $\mathrm{Lg} \mathrm{BNP}$, there was a significantly increased contribution of $\mathrm{CysC}>0.93 \mathrm{mg} / \mathrm{dl}$ to all-cause death $(\mathrm{OR}=4.059,95 \% \mathrm{Cl}: 1.045-14.340)$ in females, but showed no significance for males. Whereas referring to $\mathrm{CysC} \leq 0.93 \mathrm{mg} / \mathrm{dl}, \mathrm{CysC}>0.93 \mathrm{mg} / \mathrm{dl}$ did not show obvious links to severe inflammatory, liver injury, and heart failure both in males and females, (Fig. 2).

\section{The association between CysC rangeability and all-cause death classified by T2DM}

Compared with patients with CysC rangeability $\leq 0$, those with $\mathrm{Cys} C$ rangeability $>0$ were highly correlated with all-cause death $(\mathrm{OR}=4.029,95 \% \mathrm{Cl}: 1.864-8.706)$. For T2DM, there was a significantly increased contribution of CysC rangeability $>0$ to all-cause death (OR $=5.585,95 \% \mathrm{Cl}: 2.328-13.397)$, (Fig. 3 ).

As for the sex subgroup of T2DM patients, adjusted by age, severe pneumonia, serum albumin, blood glucose, $\mathrm{LgBNP}$, there was a significant contribution of $\mathrm{CysC}$ rangeability $>0$ to all-cause death both males $(\mathrm{OR}=4.699,95 \% \mathrm{Cl}: 1.604-13.767)$. Notably, the association increased significantly for female patients $(\mathrm{OR}=13.514,95 \% \mathrm{Cl}: 1.398-30.675)$, (Fig. 4).

\section{Discussion}




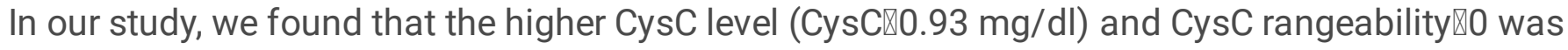
independently associated with mortality among COVID-19-associated hospitalized adults with T2DM, and CysC rangeability seems to contribute more risk for hospitalized death than a single CysC level. More importantly, our study also suggests that sex could affect this association, when classified by sex, this association is stronger in female patients. Notably, we also observed these associations in patients with generally mild pneumonia, and all case of death was contributed by T2DM.

To the best of our knowledge, there was no evidence to confirm the association between CysC and allcause death of COVID-19-associated hospitalized adults and as well as the T2DM and gender's hybrid effect in present studies. CysC, the endogenous biomarkers of renal function, can indirectly assess glomerular filtration rate (GFR) ${ }^{15}$, is produced in nucleated cells, and is uneasily influenced by decreased muscle mass, acute and chronic illness, and nutritional status than creatinine ${ }^{16}$. Moreover, over the past decade, serum CysC has been extensively studied and found to be a sensitive serum marker of the GFR and a stronger predictor than creatinine as a risk factor of cardiovascular events and all-cause death in generally older patients ${ }^{16}$. Some studies suggested that high levels of serum CysC were associated with severity and poor prognosis of cardiovascular disease ${ }^{17}$, neurodegeneration ${ }^{18}, \mathrm{CKD}^{19}$, acute kidney injury ${ }^{20}$, chronic liver disease ${ }^{21}$, critically ill intensive care unit (ICU) patients ${ }^{22}$, as well as the general population ${ }^{23}$. Thus, CysC may be a serum molecular marker for predicting poor prognosis of various acute and chronic diseases. Since the COVID-19 outbreak, more and more research has been devoted to discovering its clinical features and organ damage. Some studies have shown that heart, liver, and renal function are impaired in novel coronavirus infected patients ${ }^{8,24-26}$. What's more, the outcome of COVID19 patients is associated with organ damage. An observative study from South Korea showed that COVID-19 patients with severe AKI had fatal outcomes including death ${ }^{27}$. Those studies only observed renal function injury based on creatinine urea, but not noted the more stable biomarker of CysC. To the best of our knowledge, our study is the first to focus on CysC and CysC rangeability to observe the impact on organ function and mortality of COVID-19 patients.

Researchers have also observed that novel coronavirus- COVID-19 can directly infect the kidney and cause renal impairment. In Victor G' studies, SARS-CoV-2 viral load in organs and tissues were first quantified, of which autopsy samples obtained from 22 patients who had died from COVID-19, and found that the highest levels of SARS-CoV-2 copies per cell were detected in the respiratory tract, and lower levels in kidney, heart, liver, brain, and blood. These findings indicate kidneys are among the most common targets of SARS-CoV- $2^{28}$. RNA enrichment for transmembrane serine protease 2 (TMPRSS2), angiotensin-converting enzyme 2 (ACE2), and cathepsin L (CTSL) are considered to facilitate SARSCoV-2 infection. Whereas that RNA of genes are enriched in multiple kidney-cell types from fetal development through adulthood. This enrichment may promote SARS-CoV-2-associated kidney injury ${ }^{29,30}$. CysC is recognized as one of the most reliable renal function parameters in the general population. Thus, when Infected with novel Coronavirus- COVID-19, kidney injury can be reflected in the level of CysC. A recent study concerned about imaging progression on chest CT at the first week and CysC levels from COVID-19 patients and showed that patients with higher CysC levels with COVID-19 showed more progressive 
lesions in lung CT, in which only the predictive value of CysC level at admission to the disease progression was observed, rather than dynamic changes ${ }^{31}$. And the severity of COVID-19 is closely related to death. Our study not only found that CysC levels were associated with death in hospital from COVID-19 but also observed the changes in CysC levels during hospitalization were more significant in predicting death.

We also found that T2DM more easily caused deaths in our study population, which were consistent with the previous research that T2DM was not only associated with suffering COVID-19 but also an independent risk factor for severe pneumonia and poor prognosis ${ }^{32}$. T2DM can be defined as the phenotype of hyperglycemia that manifests a group of common metabolic disorders. The metabolic disorder related to T2DM causes secondary pathophysiological changes of multiple organ tissues and results in various complications, which contribute to the morbidity and mortality associated with the $\mathrm{T} 2 \mathrm{DM}^{33}$. The pathophysiology of SARS-CoV-2 remains incompletely understood, but evidence demonstrates that SARS-CoV-2 can trigger hyper inflammation in certain organ and emerges tissue tropism, which shared with the same pathologies for chronic inflammation and multi-organ damage in $\mathrm{T}_{2} \mathrm{DM}^{33}$. Although the intensely acute response of hyperglycemic, inflammatory, and tissue damage in SARS-CoV-2 infection, it is still mirrored by T2DM pathophysiology, which characterized by impaired glycemic regulation, chronic, low-grade inflammation condition, and slowly progressive multi-organ damage, diabetic microvascular (neuropathy, brain, chronic kidney diseases) and macrovascular (cerebrovascular disease) complications. Acute COVID-19-associated adverse response may overlay on glucose instability, preexisting inflammation, and multi-organ damages in T2DM patients, eventually aggravate outcomes ${ }^{33}$. The level of CysC increased in COVID-19 infected patients complicated with acute kidney injury, which also would be an indicator for early detection of diabetic nephropathy in type 2 T2DM. Thus Monitoring changes in CysC may be important for assessing the progression of COVID-19 patients. In the gender subgroup analysis of diabetes, CysC levels at admission were strongly associated with death in females but not for males. Although the relationship between $\mathrm{Cys} C$ rangeability and death was not sex-specific, a greater contribution was observed in women than in men, possibly because the levels of CysC were statistically significantly higher in males, compared to age-matched females ${ }^{34}$, and when males and females have the same level of $\mathrm{CysC}$, women may tend to have worse kidney function.

Because of this emergent situation, there is no sufficient report and diagnosis of chronic renal disease and other comorbidities disease. Previous studies have shown that SARS-CoV-2 may aggravate kidney injury in patients with chronic kidney diseases ${ }^{35}$. Therefore, renal injury in patients with chronic renal disease needs to be investigated and monitored. Limitations exist in our research. First, it shares all the limitations of a single-center observational study, so the generalizability of our results may be limited. This study was retrospective, and all cases did not have sufficient history of illness. The multiple tests for renal function were carried out at different time intervals for each patient. Bias may occur due to the increased number of tests in patients with renal injury.

\section{Conclusion}


In summary, CysC contributes to clinical manifestations and may influence the progression and prognosis of COVID-19 patients, with an increased risk of severe complications and death for subjects with T2DM. Thereby, more attention needs to be paid to patients with a high-level CysC as well as CysC rangeability in clinical treatment and later clinical follow-up, especially for those with T2DM. However, the exact mechanisms underlying CysC -related changes in the course of COVID-19 are not established yet and needs to be resolved in the future.

\section{Declarations}

\section{Data availability statements}

The datasets used and/or analysed during the current study are available from the corresponding author on reasonable request.

\section{Ethics approval and consent to participate}

The study protocol complies with the Declaration of Helsinki and was approved by the Institutional Review Board of the Wuhan Third Hospital Foundation. Because of the study's retrospective design, informed consent was waived in cases of discharge, of impossibility of contact with patients and in case of death. The privacy and anonymity of the data collected was guaranteed in accordance with current regulations.

\section{Competing interests}

The authors declare that they have no competing interests.

\section{Consent for publication}

All authors consent to the publication.

\section{Funding}

This work is partially supported by the National Natural Science Foundation of China under Grant No. 61902310, and Fundamental Research Funds for Central Universities under Grant Nos. xjh012019039.

\section{Authors' contributions}

Lei Yang, Dou Xu and Yiqing Tan contributed equally to the study and manuscript. Lei Yang, Dou Xu and Yiqing Tan contributed to the conception and design of the study, analysis and interpretation of the data, wrote the manuscript, and approved submission. Bolin Li contributed to the data acquisition and analysis. Dan Zhu, Jingbo Wang and Hui Sun collected the data. Xinglong Liu and Xiaopu Zheng contributed to analysis of data and provided critical revision of the paper. Ling Zhu and Zhongyu Li provided critical revision of the paper for important intellectual content. All authors read and approved the fnal manuscript. 
Acknowledgements

Not applicable.

\section{References}

1. Acter T, Uddin N, Das J, Akhter A, Choudhury TR, Kim S. Evolution of severe acute respiratory syndrome coronavirus 2 (SARS-CoV-2) as coronavirus disease 2019 (COVID-19) pandemic: A global health emergency. Sci Total Environ. 2020;730:138996.

2. Li Q, Guan X, Wu P, Wang X, Zhou L, Tong Y, Ren R, Leung KSM, Lau EHY, Wong JY, et al. Early Transmission Dynamics in Wuhan, China, of Novel Coronavirus-Infected Pneumonia. N Engl J Med. 2020;382(13):1199-1207.

3. Wu JT, Leung K, Leung GM. Nowcasting and forecasting the potential domestic and international spread of the 2019-nCoV outbreak originating in Wuhan, China: a modelling study. Lancet. 2020;395(10225):689-697.

4. Tu H, Tu S, Gao S, Shao A, Sheng J. Current epidemiological and clinical features of COVID-19; a global perspective from China. $J$ Infect. 2020;81(1):1-9.

5. Chen C, Chen C, Yan JT, Zhou N, Zhao JP, Wang DW. [Analysis of myocardial injury in patients with COVID-19 and association between concomitant cardiovascular diseases and severity of COVID-19]. Zhonghua Xin Xue Guan Bing Za Zhi. 2020;48(7):567-571.

6. Huang C, Wang Y, Li X, Ren L, Zhao J, Hu Y, Zhang L, Fan G, Xu J, Gu X, et al. Clinical features of patients infected with 2019 novel coronavirus in Wuhan, China. Lancet. 2020;395(10223):497-506.

7. Zaim S, Chong JH, Sankaranarayanan V, Harky A. COVID-19 and Multiorgan Response. Curr Prob/ Cardiol. 2020;45(8):100618-100618.

8. Gabarre P, Dumas G, Dupont T, Darmon M, Azoulay E, Zafrani L. Acute kidney injury in critically ill patients with COVID-19. Intensive Care Med. 2020;46(7):1339-1348.

9. Fu L, Fei J, Xu S, Xiang HX, Xiang Y, Hu B, Li MD, Liu FF, Li Y, Li XY, et al. Liver Dysfunction and Its Association with the Risk of Death in COVID-19 Patients: A Prospective Cohort Study. J Clin Transl Hepatol. 2020; 8(3):246-254.

10. Cheng Y, Luo R, Wang K, Zhang M, Wang Z, Dong L, Li J, Yao Y, Ge S, Xu G. Kidney disease is associated with in-hospital death of patients with COVID-19. Kidney Int. 2020;97(5):829-838.

11. Hong XW, Chi ZP, Liu GY, Huang H, Guo SQ, Fan JR, Lin XW, Qu LZ, Chen RL, Wu LJ, et al. Characteristics of Renal Function in Patients Diagnosed With COVID-19: An Observational Study. Front Med (Lausanne). 2020;7:409.

12. Uribarri A, Nunez-Gil IJ, Aparisi A, Becerra-Munoz VM, Feltes G, Trabattoni D, Fernandez-Rozas I, Viana-Llamas MC, Pepe M, Cerrato E, et al. Impact of renal function on admission in COVID-19 patients: an analysis of the international HOPE COVID-19 (Health Outcome Predictive Evaluation for COVID 19) Registry. J Nephrol. 2020;33(4):737-745. 
13. Cheng Y, Luo R, Wang K, Zhang M, Wang Z, Dong L, Li J, Yao Y, Ge S, Xu G. Kidney disease is associated with in-hospital death of patients with COVID-19. Kidney Int. 2020;97(5):829-838.

14. Wang X, Wang S, Sun L, Qin G. Prevalence of diabetes mellitus in 2019 novel coronavirus: A metaanalysis. Diabetes Res Clin Pract. 2020;164:108200.

15. Carlier M, Dumoulin A, Janssen A, Picavet S, Vanthuyne S, Van Eynde R, Vanholder R, Delanghe J, De Schoenmakere G, De Waele JJ, et al. Comparison of different equations to assess glomerular filtration in critically ill patients. Intensive Care Med. 2015;41(3):427-435.

16. Kashani KB, Frazee EN, Kukrálová L, Sarvottam K, Herasevich V, Young PM, Kashyap R, Lieske JC. Evaluating Muscle Mass by Using Markers of Kidney Function: Development of the Sarcopenia Index. Crit Care Med. 2017;45(1):e23-e29.

17. Luo J, Wang L, Hu H, Zhang L, Li Y, Ai L, Mu H, Kun-Wang. Cystatin C and cardiovascular or all-cause mortality risk in the general population: A meta-analysis. Clin Chim Acta. 2015;450:39-45.

18. Gauthier S, Kaur G, Mi W, Tizon B, Levy E. Protective mechanisms by cystatin C in neurodegenerative diseases. Front Biosci (Schol Ed). 2011;3:541-554.

19. Bevc S, Hojs N, Knehtl M, Ekart R, Hojs R. Cystatin C as a predictor of mortality in elderly patients with chronic kidney disease. Aging Male. 2019;22(1):62-67.

20. Maiwall R, Kumar A, Bhardwaj A, Kumar G, Bhadoria A, Sarin S. Cystatin C predicts acute kidney injury and mortality in cirrhotics: A prospective cohort study. Liver Int. 2018;38(4):654-664.

21. Uguen T, Jezequel C, Ropert M, Houssel-Debry P, Latournerie M, Lainé F, Deugnier Y, Vigneau C, Boudjema K, Guyader D, Bardou-Jacquet E. Pretransplant renal function according to CKD-EPI cystatin $\mathrm{C}$ equation is a prognostic factor of death after liver transplantation. Liver Int. 2016;36(4):547-554.

22. Ravn B, Prowle J, Mårtensson J, Martling C, Bell M. Superiority of Serum Cystatin C Over Creatinine in Prediction of Long-Term Prognosis at Discharge From ICU. Crit Care Med. 2017;45(9):e932-e940.

23. Helmersson-Karlqvist J, Ärnlöv J, Larsson A. Cystatin C-based glomerular filtration rate associates more closely with mortality than creatinine-based or combined glomerular filtration rate equations in unselected patients. Eur J Prev Cardiol. 2016;23(15):1649-1657.

24. Phipps MM, Barraza LH, LaSota ED, Sobieszczyk ME, Pereira MR, Zheng EX, Fox AN, Zucker J, Verna EC. Acute Liver Injury in COVID-19: Prevalence and Association with Clinical Outcomes in a Large US Cohort. Hepatology. 2020.

25. Puelles VG, Lütgehetmann M, Lindenmeyer MT, Sperhake JP, Wong MN, Allweiss L, Chilla S, Heinemann A, Wanner N, Liu S, et al. Multiorgan and Renal Tropism of SARS-CoV-2. N Engl J Med. 2020;383(6):590-592.

26. Shi S, Qin M, Cai Y, Liu T, Shen B, Yang F, Cao S, Liu X, Xiang Y, Zhao Q, et al. Characteristics and clinical significance of myocardial injury in patients with severe coronavirus disease 2019. Eur Heart J. 2020;41(22):2070-2079.

27. Lim JH, Park SH, Jeon Y, Cho JH, Jung HY, Choi JY, Kim CD, Lee YH, Seo H, Lee J,et al. Fatal Outcomes of COVID-19 in Patients with Severe Acute Kidney Injury. J Clin Med. 2020;9(6). 
28. Puelles V, Lütgehetmann M, Lindenmeyer M, Sperhake J, Wong M, Allweiss L, Chilla S, Heinemann A, Wanner N, Liu S,et al. Multiorgan and Renal Tropism of SARS-CoV-2. N Engl J Med. 2020;383(6):590592.

29. Sungnak W, Huang N, Bécavin C, Berg M, Queen R, Litvinukova M, Talavera-López C, Maatz H, Reichart D, Sampaziotis F, et al. SARS-CoV-2 entry factors are highly expressed in nasal epithelial cells together with innate immune genes. Nat Med. 2020;26(5):681-687.

30. Zhang YM, Zhang H. Genetic Roadmap for Kidney Involvement of Severe Acute Respiratory Syndrome Coronavirus 2 (SARS-CoV-2) Infection. Clin J Am Soc Nephrol. 2020;15(7):1044-1046.

31. Yang Z, Shi J, He Z, Lü Y, Xu Q, Ye C, Chen S, Tang B, Yin K, Lu Y,et al. Predictors for imaging progression on chest CT from coronavirus disease 2019 (COVID-19) patients. Aging. 2020;12(7):6037-6048.

32. Feldman E, Savelieff M, Hayek S, Pennathur S, Kretzler M, Pop-Busui R. COVID-19 and Diabetes: A Collision and Collusion of Two Diseases. Diabetes. 2020.

33. Feldman EL, Savelieff MG, Hayek SS, Pennathur S, Kretzler M, Pop-Busui R. COVID-19 and Diabetes: A Collision and Collusion of Two Diseases. Diabetes. 2020.

34. Marmarinos A, Garoufi A, Panagoulia A, Dimou S, Drakatos A, Paraskakis I, Gourgiotis D. Cystatin-C levels in healthy children and adolescents: Influence of age, gender, body mass index and blood pressure. Clin Biochem. 2016;49:150-153.

35. Wang Y, LV Y, Liu Q. SARS-CoV-2 infection associated acute kidney injury in patients with pre-existing chronic renal disease: A report of two cases. Immun Inflamm Dis. 2020.

\section{Figures}



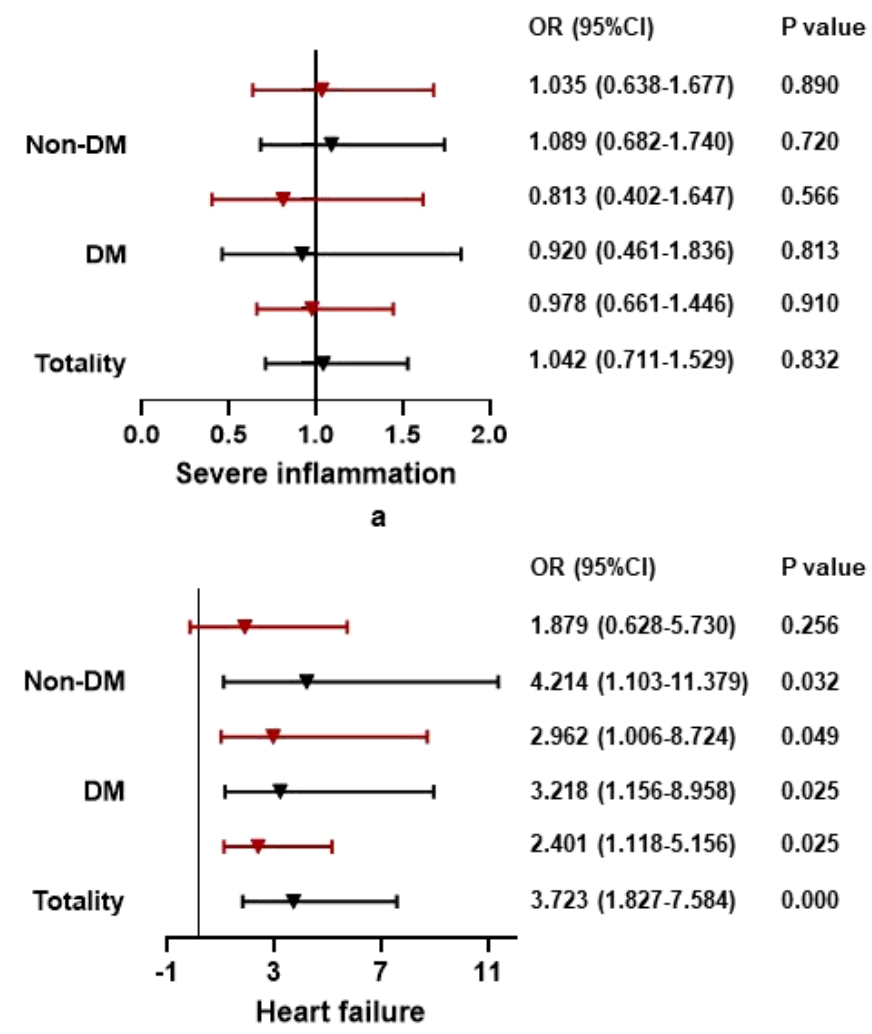

c

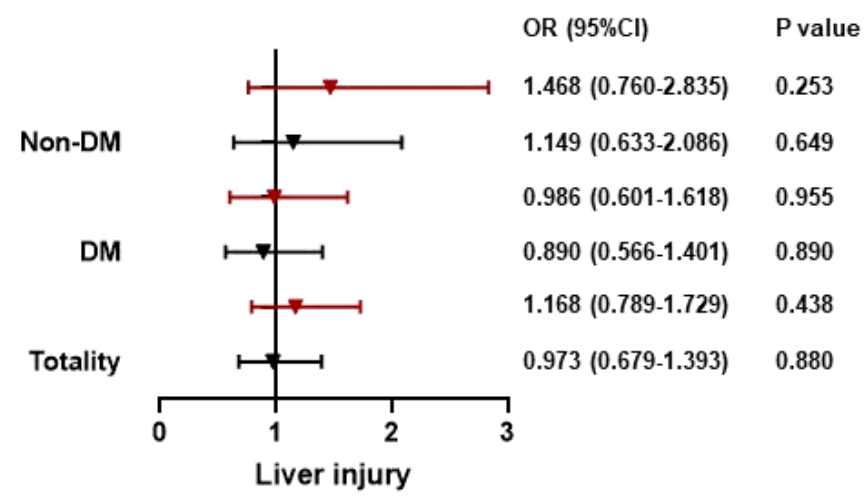

b

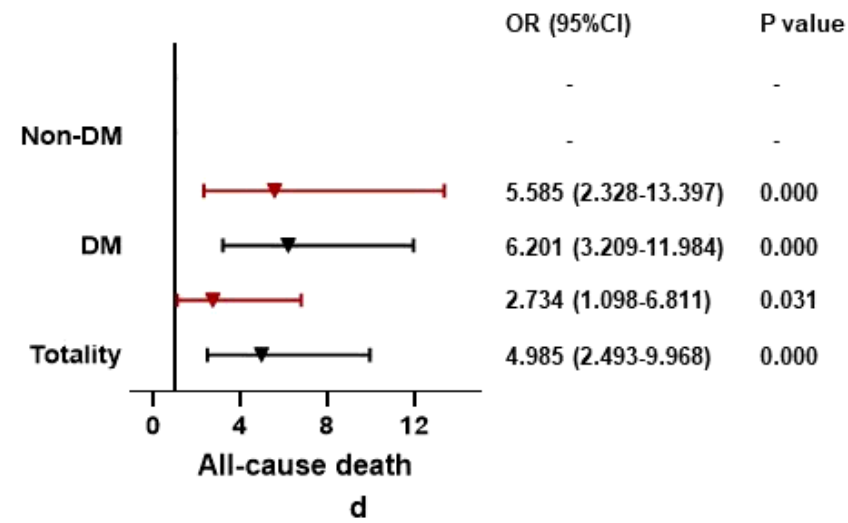

Figure 1

\section{Figure 1}

The association between CysC and organ dysfunction, all-cause death classified by diabetes mellitus; $†$ a, the association between $\mathrm{Cys} C$ and severe inflammation classified by diabetes mellitus; $b$, the association between CysC and liver injury classified by diabetes mellitus; $c$, the association between CysC and heart failure classified by diabetes mellitus; $d$, the association between CysC and all-cause death classified by diabetes mellitus; ¥ Severe inflammation was defined as highest NLR during hospitalization higher than 10. Liver injury was defined as ALT or AST at any time during hospitalization greater than 80U/L. Heart failure was defined as highest BNP during hospitalization greater than $300 \mathrm{pg} / \mathrm{ml}$. $\S$ Adjusted by age, severe pneumonia, serum albumin, blood glucose, Lg BNP baseline. 9 DM, diabetes mellitus; CysC, cystatin C; NLR囚Neutrophil-to-Lymphocyte Ratio; ALT, Alanine aminotransferase; AST, Aspartate aminotransferase; BNP, Brain Natriuretic Peptide. 

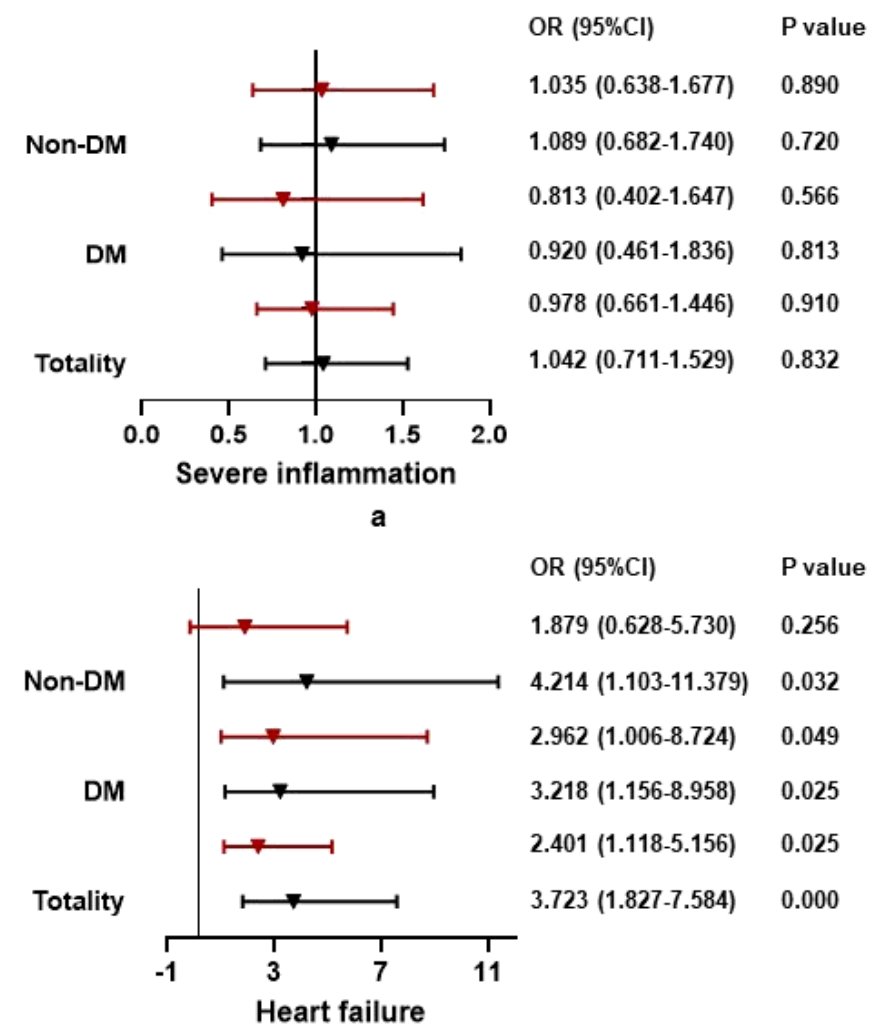

c

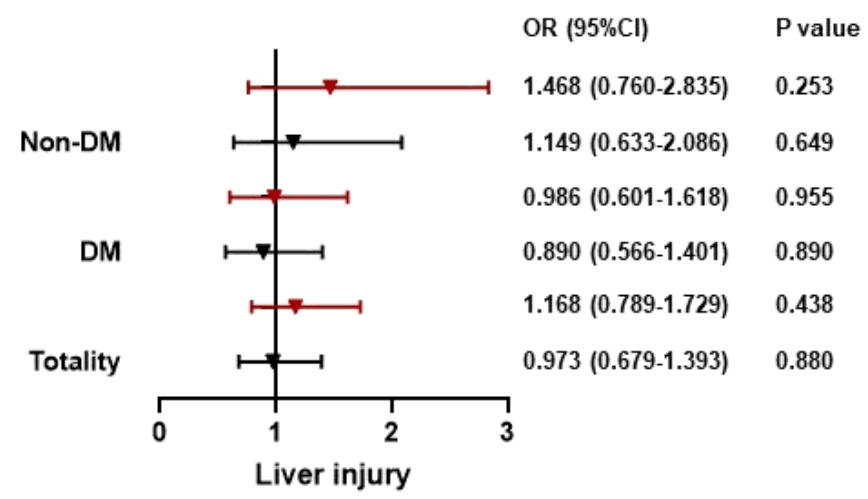

b

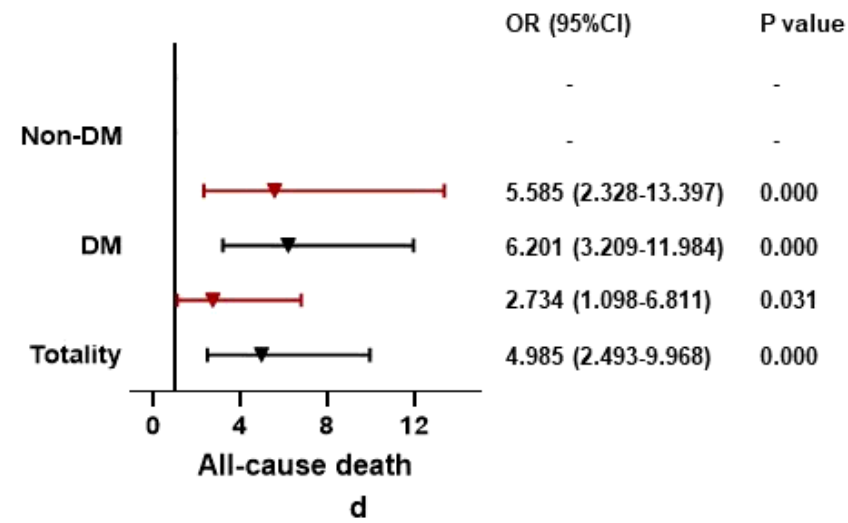

Figure 1

\section{Figure 1}

The association between CysC and organ dysfunction, all-cause death classified by diabetes mellitus; $†$ a, the association between $\mathrm{Cys} C$ and severe inflammation classified by diabetes mellitus; $b$, the association between CysC and liver injury classified by diabetes mellitus; $c$, the association between CysC and heart failure classified by diabetes mellitus; $d$, the association between CysC and all-cause death classified by diabetes mellitus; ¥ Severe inflammation was defined as highest NLR during hospitalization higher than 10. Liver injury was defined as ALT or AST at any time during hospitalization greater than 80U/L. Heart failure was defined as highest BNP during hospitalization greater than $300 \mathrm{pg} / \mathrm{ml}$. $\S$ Adjusted by age, severe pneumonia, serum albumin, blood glucose, Lg BNP baseline. 9 DM, diabetes mellitus; CysC, cystatin C; NLR囚Neutrophil-to-Lymphocyte Ratio; ALT, Alanine aminotransferase; AST, Aspartate aminotransferase; BNP, Brain Natriuretic Peptide. 


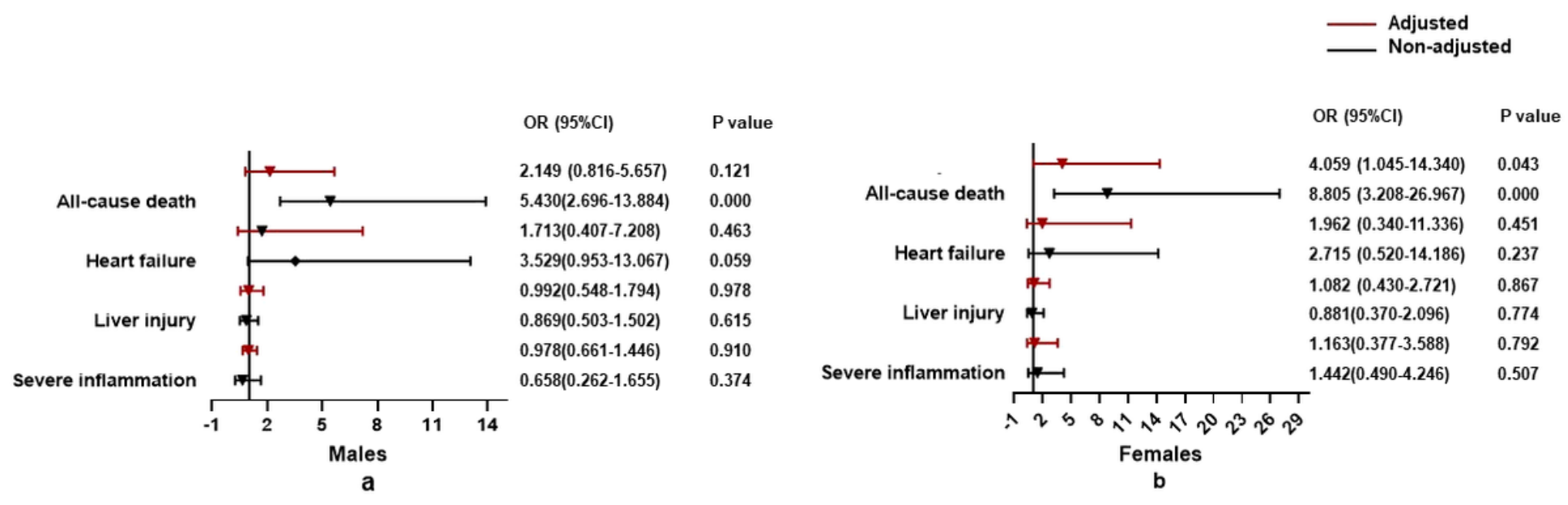

Figure 2

Figure 2

The association between CysC and organ dysfunction, all-cause death in diabetes population classified by sex. $\uparrow a$, the association between $\mathrm{CysC}$ and organ dysfunction, all-cause death in male diabetes population; $b$, the association between CysC and in female diabetes population. $¥$ Severe inflammation was defined as highest NLR during hospitalization higher than 10. Liver injury was defined as ALT or AST at any time during hospitalization greater than $80 \mathrm{U} / \mathrm{L}$. Heart failure was defined as highest BNP during hospitalization greater than $300 \mathrm{pg} / \mathrm{ml}$. § Adjusted by age, severe pneumonia, serum albumin, blood glucose, Lg BNP baseline. 9 DM, diabetes mellitus; CysC, cystatin C; NLR囚Neutrophil-to-Lymphocyte Ratio; ALT, Alanine aminotransferase; AST, Aspartate aminotransferase; BNP, Brain Natriuretic Peptide.

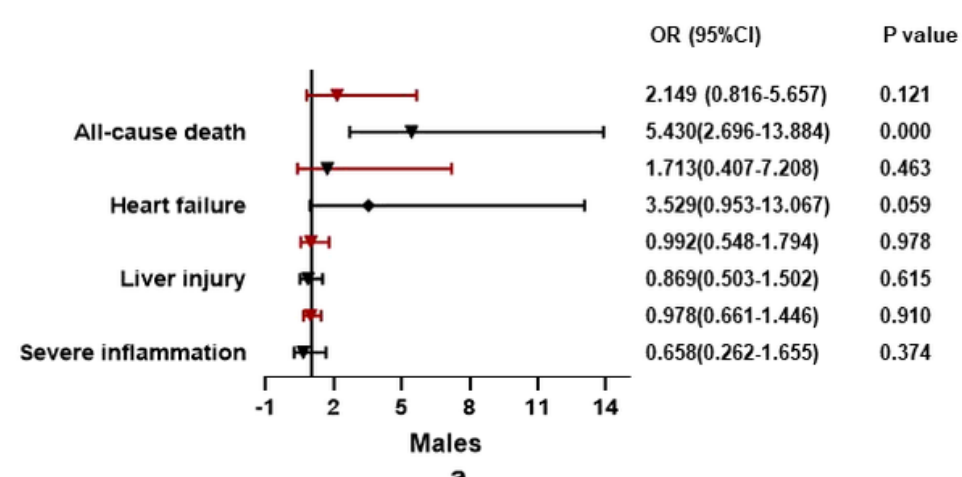

Figure 2

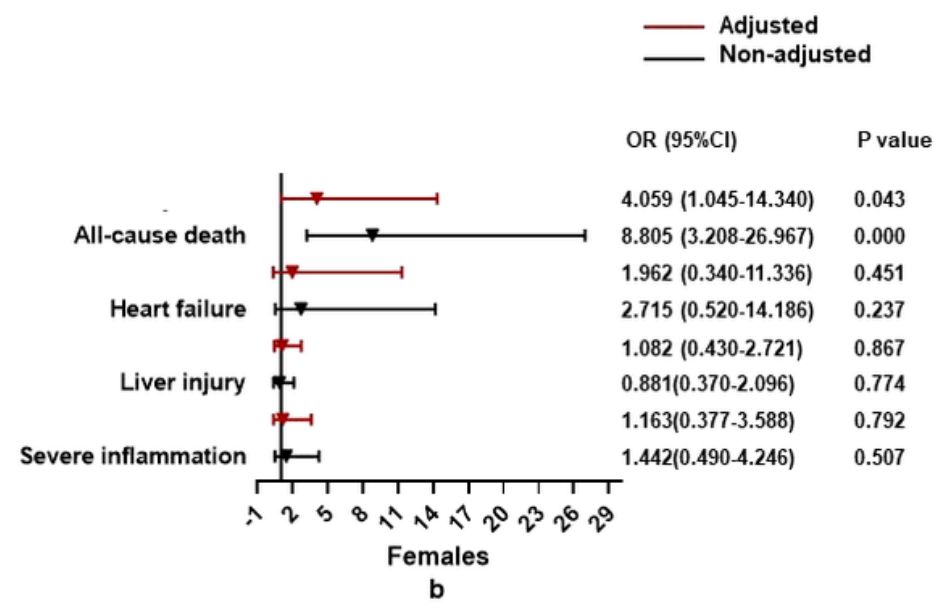

\section{Figure 2}

The association between CysC and organ dysfunction, all-cause death in diabetes population classified by sex. $+\mathrm{a}$, the association between $\mathrm{CysC}$ and organ dysfunction, all-cause death in male diabetes population; $b$, the association between CysC and in female diabetes population. ¥ Severe inflammation was defined as highest NLR during hospitalization higher than 10. Liver injury was defined as ALT or AST at any time during hospitalization greater than $80 \mathrm{U} / \mathrm{L}$. Heart failure was defined as highest BNP during 
hospitalization greater than $300 \mathrm{pg} / \mathrm{ml}$. $\S$ Adjusted by age, severe pneumonia, serum albumin, blood glucose, Lg BNP baseline. 9 DM, diabetes mellitus; CysC, cystatin C; NLR『Neutrophil-to-Lymphocyte Ratio; ALT, Alanine aminotransferase; AST, Aspartate aminotransferase; BNP, Brain Natriuretic Peptide.

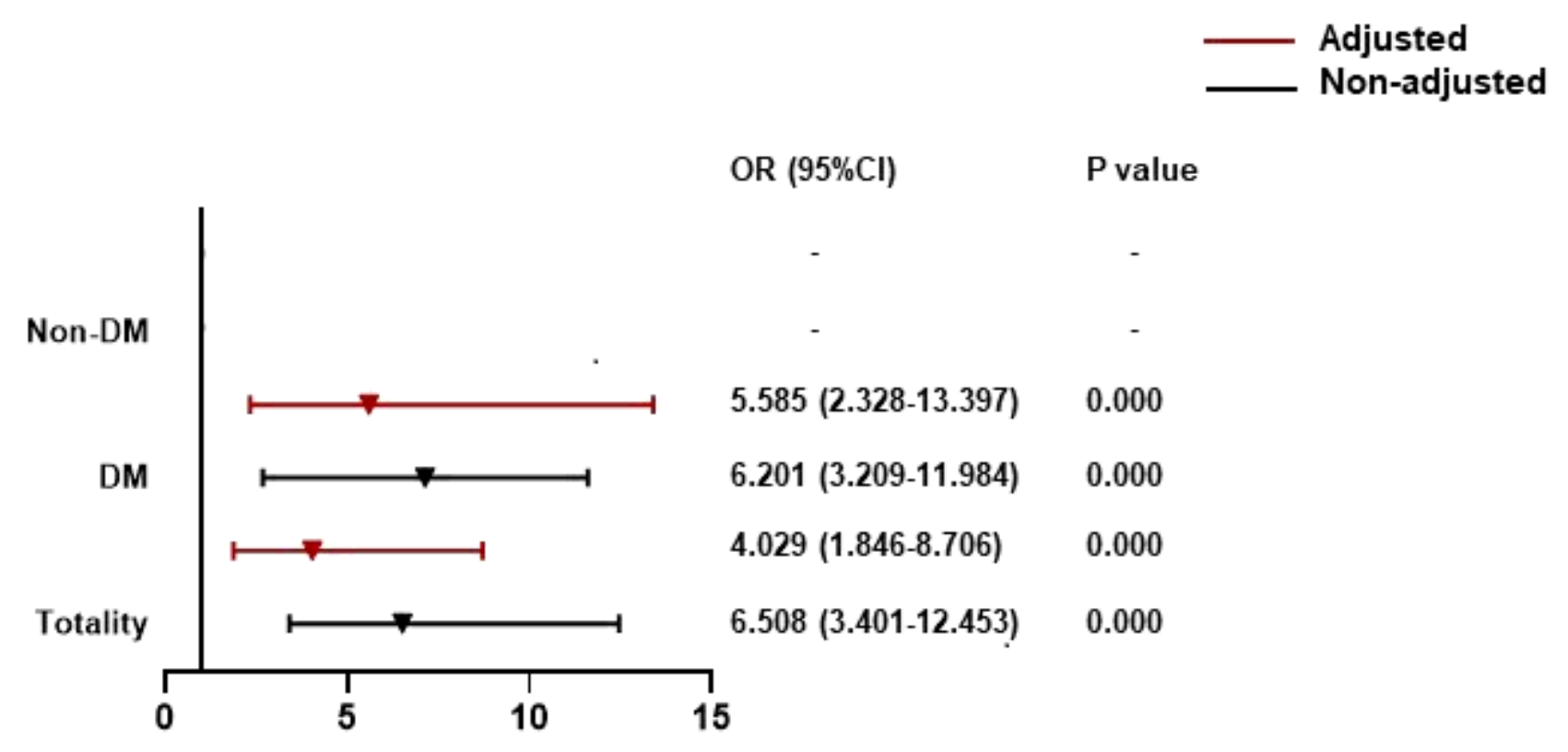

\section{Figure 3}

\section{Figure 3}

The association between CysC rangeability and all-cause death classified by DM. + CysC rangeability (renal function changes) calculated as the difference of $\mathrm{CysC}$ level between the time of admission and the highest CysC level testing during hospitalization. $¥$ Adjusted by age, severe pneumonia, serum albumin, blood glucose, Lg BNP baseline. §̧DM, diabetes mellitus; BNP, Brain Natriuretic Peptide. 


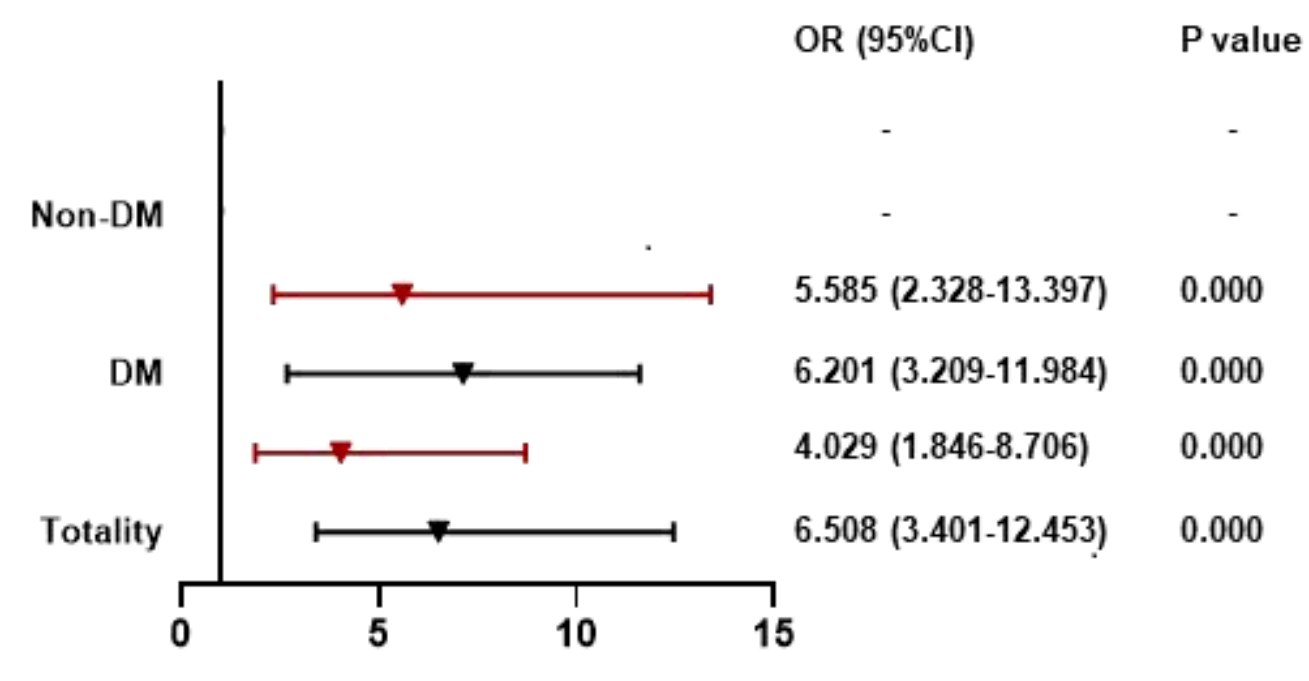

Figure 3

Figure 3

The association between CysC rangeability and all-cause death classified by DM. + CysC rangeability (renal function changes) calculated as the difference of CysC level between the time of admission and the highest CysC level testing during hospitalization. $¥$ Adjusted by age, severe pneumonia, serum albumin, blood glucose, Lg BNP baseline. §̧DM, diabetes mellitus; BNP, Brain Natriuretic Peptide.

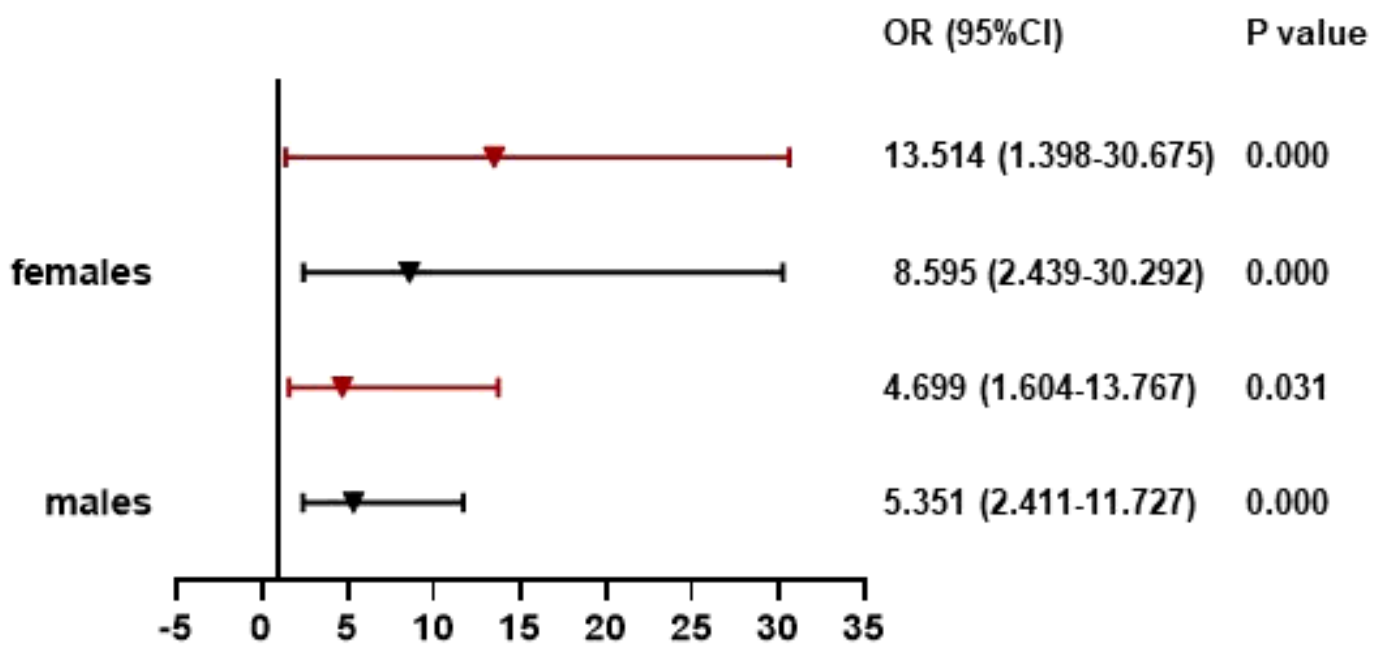

Figure 4

Figure 4 
The association between CysC rangeability and all-cause death in $\mathrm{DM}$ population. + CysC rangeability (renal function changes) calculated as the difference of CysC level between the time of admission and the highest CysC level testing during hospitalization. $¥$ Adjusted by age, severe pneumonia, serum albumin, blood glucose, Lg BNP baseline. §̧DM, diabetes mellitus; BNP, Brain Natriuretic Peptide.

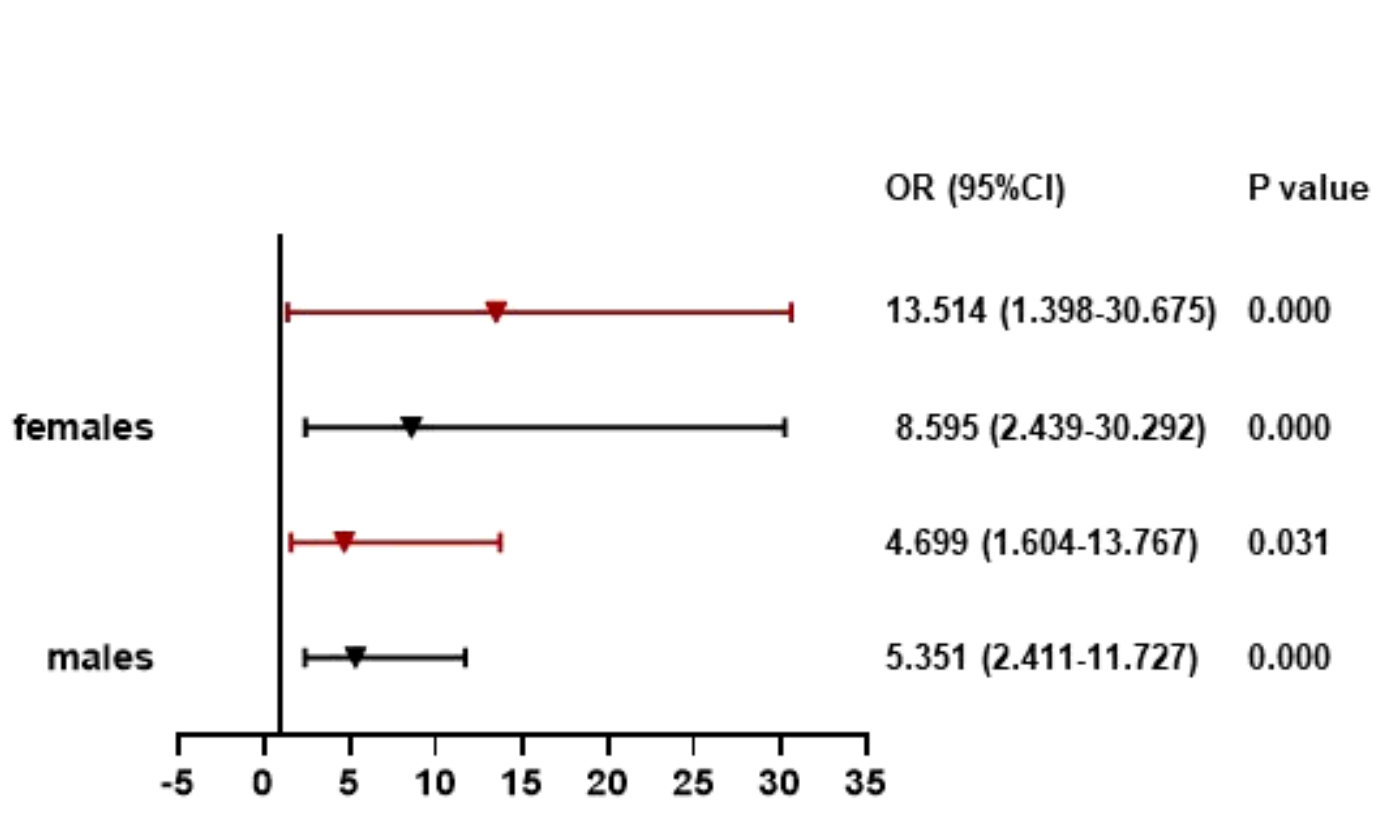

\section{Figure 4}

\section{Figure 4}

The association between CysC rangeability and all-cause death in $\mathrm{DM}$ population. $+\mathrm{Cys} \mathrm{C}$ rangeability (renal function changes) calculated as the difference of $\mathrm{CysC}$ level between the time of admission and the highest CysC level testing during hospitalization. $¥$ Adjusted by age, severe pneumonia, serum albumin, blood glucose, Lg BNP baseline. §DM, diabetes mellitus; BNP, Brain Natriuretic Peptide. 